\title{
El aprendizaje basado en la investigación en el contexto universitario: una revisión sistemática*
}

\section{Inquiry-based learning in the university context: A systematic review}

\author{
Dra. Lidia E. SANTANA.VEGA. Catedrática. Universidad de La Laguna (Isantana@ull.es). \\ Dra. Arminda SUÁREZ-PERDOMO. Profesora Contratada Doctora. Universidad de La Laguna (asuper@ull.edu.es). \\ Dr. Luis FELICIANO-GARCÍA. Profesor Titular. Universidad de La Laguna (Ifelici@ull.edu.es).
}

\section{Resumen:}

El aprendizaje basado en la investigación (ABI) es una metodología que potencia los aprendizajes mediante un proceso de construcción del conocimiento. La finalidad del estudio era conocer cómo se está aplicando el ABI y sus efectos en estudiantes universitarios de ciencias sociales y de la salud. El método para realizar la revisión sistemática ha seguido las directrices de la declaración PRISMA. Se analizaron un total de 31 estudios extraídos de cuatro bases de datos electrónicas y listas de referencias sobre el tópico, publicados en inglés entre 1998-2019. Los resultados mostraron como fortalezas del ABI: 1) la promoción del aprendizaje coo- perativo; 2) el compromiso del alumnado en su autoaprendizaje; y, 3) el aumento de un pensamiento crítico. Entre sus debilidades se señalan: 1) la incapacidad de cubrir las expectativas de aprendizaje, y 2) las reticencias de las estructuras universitarias hacia el ABI. A partir de estos resultados se discute el valor del ABI como estrategia didáctica en la universidad, en la medida en que permite profundizar en la construcción del conocimiento, incrementar la motivación de aprendizaje, desarrollar las habilidades de investigación, el autoaprendizaje, la autoconfianza, el pensamiento crítico, y el rendimiento académico. El ABI favorece el aprendizaje significativo en el alumnado universitario al ofrecer un espacio

\footnotetext{
* Este trabajo surge de un proyecto más amplio financiado por la Comisión Europea (Education, Audiovisual and Culture Executive Agency -EACEA-), REFLECT LAB: Apoyo al profesorado en la aplicación del aprendizaje basado en la investigación (2016-DE01-KA203-002891). Erasmus+Asociaciones Estratégicas en el ámbito de la Educación Superior. Esta publicación refleja solo los puntos de vista de los autores, la Comisión no se hace responsable del uso que pueda hacerse de la información contenida en el mismo.
}

Fecha de recepción de la versión definitiva de este artículo: 22-06-2020.

Cómo citar este artículo: Santana-Vega, L. E., Suárez-Perdomo, A., Feliciano-García, L. (2020). El aprendizaje basado en la investigación en el contexto universitario: una revisión sistemática | Inquiry-based learning in the university context: A systematic review. Revista Española de Pedagogía, 78 (277), 519-537. doi: https://doi.org/10.22550/REP78-3-2020-08 https://revistadepedagogia.org/

ISSN: 0034-9461 (Impreso), 2174-0909 (Online) 
de creación de conocimiento estimulado por el proceso de indagación.

Descriptores: aprendizaje basado en la investigación, alumnado universitario, métodos de enseñanza, aprendizaje activo, revisión sistemática, PRISMA.

\section{Abstract:}

Inquiry-Based Learning (IBL) is a methodology that enhances learning through a knowledge construction process. The aim of this study is to establish how IBL is used and what effects it has on university students from social sciences and health sciences. This study follows the PRISMA guidelines for conducting systematic reviews. It comprises an analysis of 31 studies extracted from four electronic databases and reference lists on the topic, published in English between 1998-2019. The results show that the strengths of IBL are: 1) promoting cooperative learning, 2) engaging students in self-learning, and 3) increasing critical thinking. Its weaknesses include: 1) the inability to meet learning expectations, and 2) the reluctance of university hierarchies to embrace IBL. The potential of IBL as a teaching strategy at university level is discussed as it allows deep knowledge construction, increased learning motivation, and development of students' research skills as well as their self-learning, self-confidence, critical thinking, and academic performance. IBL favours meaningful learning by university students by offering a space for the creation of knowledge stimulated by the inquiry process.

Keywords: inquiry-based learning, college students, teaching methods, active learning, systematic review, PRISMA.

\section{Introducción}

El aprendizaje basado en la investigación (ABI) engloba una variedad de enfoques pedagógicos procedentes de los postulados de Dewey y Bruner (Herman y Pinard, 2015); según estos autores la indagación está en el centro de las tareas, en el uso de recursos, y en las instrucciones de aprendizaje. A través del ABI se propone a los estudiantes desafíos que: a) catalizan su compromiso y participación; b) fomentan un aprendizaje experiencial; $y$, c) estimulan la exploración y la búsqueda de soluciones (Aditomo, Goodyear, Bliuc y Ellis, 2013; Levy, Aiyegbayo y Little, 2009; Oliver, 2008; Prince y Felder, 2007; Spronken-Smith, Angelo, Matthews,
0'Steen, y Robertson, 2007). El ABI es un enfoque prometedor para mejorar el proceso de enseñanza y aprendizaje en las universidades.

El ABI promueve una pedagogía centrada en el alumnado como agente activo en la búsqueda y construcción del conocimiento (Healey y Jenkins, 2009; Justice, Rice y Warry, 2009; Sproken-Smith y Walker, 2010). La importancia de las pedagogías basadas en la investigación radica en fomentar la capacidad del estudiante de adoptar estrategias de aprendizaje por medio del uso de técnicas y herramientas de indagación, permitiendo profundizar en la construcción de su propio conoci- 
miento (Levy y Petrulis, 2012). En el ABI: a) se incorpora la indagación científica al proceso de enseñanza-aprendizaje; b) la enseñanza se centra en la persona que aprende; c) el aprendizaje es estimulado por la investigación al plantear preguntas o dudas; d) el profesorado asume un rol de facilitador del conocimiento; e) el aprendizaje es fruto de un proceso de construcción de conocimiento que fomenta la cognición y la metacognición; f) se estimula el aprendizaje autodirigido (Aditomo et al., 2013; Levy y Petrulis, 2012; Spronken-Smith y Walker, 2010; Justice et al., 2007; Kahn y 0'Rourke, 2004).

El ABI proporciona un amplio andamiaje social y asesoramiento a los estudiantes para gestionar su investigación (Hmelo-Silver, Duncan, y Chinn, 2007); potencia un aprendizaje activo con efectos positivos en los logros y las actitudes de los estudiantes hacia la investigación (Maass y Engeln, 2018); estimula la capacidad de resolver problemas, el pensamiento crítico y la reflexión sobre el aprendizaje (Bruder y Prescott, 2013; Minner, Levy y Century, 2010); fomenta la competencia investigadora y la formación de los estudiantes universitarios, mejorando la calidad de sus aprendizajes y el proceso de colaboración entre iguales (Bevins y Price, 2016); promueve en el alumnado una mayor comprensión de las materias, asumiendo los desafíos que comporta su formación académica (Åkerlind, 2008; Brew, 2003; Healey y Jenkins, 2009; Hunter, Laursen y Seymour, 2007); incrementa sus habilidades en la redacción de documentos académicos (Justice, Rice y Warry, 2009).
Griffiths (2004) y Healey (2005) señalan cuatro modalidades de $\mathrm{ABI}$ en función del modo de construir el vínculo entre la enseñanza y la investigación:

a) Research-led. El currículum está dominado por los intereses del profesorado, quien establece el modelo de transmisión de la información. Los estudiantes aprenden sobre los resultados de la investigación.

b) Research-oriented. El profesorado intenta generar una ética de la investigación a través de la enseñanza; el currículum enfatiza tanto los procesos que producen el conocimiento como el aprendizaje logrado. Los estudiantes aprenden en el proceso de investigación.

c) Research-based. La división entre los roles de estudiante y profesorado está minimizada, el currículum se diseña, en gran medida, sobre actividades basadas en la investigación. Los estudiantes aprenden como investigadores.

d) Research-tutored. Los estudiantes aprenden basándose en los resultados de la investigación, elaborados en pequeños grupos de discusión con un profesor que dinamiza el proceso.

Según Healey (2005) para hacer factible el nexo investigación-enseñanza es necesario rediseñar el currículum, planificando un proceso de enseñanza centrado en el estudiante como artífice de su propio aprendizaje. La aplicación del ABI 
se ha centrado en titulaciones de ciencias como matemáticas, física, o biología. Los estudios de revisión sistemática sobre la aplicación del ABI con alumnado universitario de titulaciones de naturaleza social y/o asistencial son escasos. A fin de conocer cómo se está aplicando el ABI en dichas titulaciones se llevó a cabo un estudio siguiendo las directrices de la declaración PRISMA para realizar revisiones sistemáticas y meta-análisis (Liberati et al., 2009). Los objetivos de este estudio son:

1. Identificar la duración, procedencia y características metodológicas de los estudios analizados.

2. Examinar el tipo de modalidad de ABI utilizada.

3. Analizar los objetivos de los estudios en relación a la aplicación del ABI y sus efectos en el alumnado de ciencias sociales y de la salud.

4. Analizar las limitaciones de los estudios.

\section{Método}

\subsection{Búsqueda y criterios de elegibilidad}

Para la búsqueda de publicaciones sobre el ABI en el alumnado universitario de ciencias sociales y de la salud, se planteó una serie de criterios de inclusión y exclusión: a) el artículo debe indicar específicamente el uso de metodologías activas como el ABI, se excluyen los artículos que solo mencionan «Research Teaching Nexus» (nexo entre la investigación y la enseñanza), "Learning Strategies» (estrategias de aprendizaje),
«Competence-Based Learning» (aprendizaje basado en competencias); b) la población objeto de estudio debe ser estudiantes universitarios, se excluyen los estudios centrados en alumnado no universitario; c) el rango de las fechas de las publicaciones incluidas en la revisión debe ser 19982019, se excluyen los artículos publicados con anterioridad; d) las publicaciones seleccionadas han de circunscribirse al ámbito de las ciencias sociales (educación, psicología, antropología, trabajo social, etc.) y ciencias de la salud (medicina, enfermería, fisioterapia, etc.), se excluyen los artículos centrados en titulaciones de otras áreas de conocimiento; e) los artículos deben estar escritos en inglés, se excluyen los redactados en otros idiomas.

La revisión sistemática se realizó utilizando diferentes bases de datos en Internet: ERIC, Web of Science, Current Contents Connect, MEDLINE, PsycArticles, Academic Search Complete y PsycInfo. Estas bases de datos fueron seleccionadas por su relevancia al contener artículos científicos publicados en revistas indexadas. Para identificar los términos de búsqueda relacionados con el tema a estudiar, se realizó una búsqueda bibliográfica preliminar y se consultó a expertos en ABI. Se realizó una búsqueda iterativa en cada base de datos, combinando los dos conjuntos de términos indicados en el Gráfico 1.

Se utilizaron términos Thesaurus (Gráfico 2) en las bases de datos que tenían esta posibilidad de búsqueda para conseguir registros con los términos exactos, según los criterios de inclusión establecidos en el estudio. 
GRÁFico 1. Estrategia de búsqueda truncada.

su(Inquiry-based learning OR Enquiry-based learning OR Guidedinquiry* OR Inquiry-based learning cycle OR Research teaching nexus OR research-based* method) AND su(method of teaching IBL* OR active learning OR Undergraduates* research OR self-directed learning OR student-centred OR student-focus OR High education)

Fuente: Elaboración propia.

GráfICo 2. Estrategia de búsqueda de términos Thesaurus.

SU.EXACT("Inquiry-based learning") OR SU.EXACT("Enquiry-based learning") OR SU.EXACT("Inquiry-based learning cycle") OR SU.EXACT("Inquiry-based activities") AND SU.EXACT("Researchled learning") OR SU.EXACT("Research-oriented learning") OR SU.EXACT("Research-based learning")

Fuente: Elaboración propia.

2.2. Procedimiento de selección y análisis de datos

Los pasos realizados en cada búsqueda fueron:

1. Establecer términos de búsqueda truncados y términos Thesaurus para delimitar las búsquedas según los objetivos del estudio.

2. Realizar la búsqueda de registros en las bases de datos seleccionadas.

3. Ordenar por relevancia los registros obtenidos. Cuando el número de búsqueda superaba los 100 registros, se utilizaron los siguientes filtros para reducir su número: estar sometidos a revisiones doble ciego; disponer de textos completos con vínculo; estar comprendidos en el rango temporal 1998-2019; y publicados en inglés en revistas académicas.

4. Realizar una segunda selección de los registros recuperados, tomando como criterio el título y/o resumen, y excluyendo aquellos que no se ajustaban al área temática.

5. Elaborar una hoja de extracción de datos para cada artículo con los criterios de inclusión especificando: referencia del estudio; motivo de selección; título y/o resumen (población universitaria, ciencias sociales o de la salud, $A B I$ ); año de publicación. Finalmente se determinó su adecuación para un posterior análisis.

6. Verificar el grado de exactitud de los datos extraídos en la selección de los artículos. 
Tras la búsqueda preliminar en cada base de datos, se observó que PsycArticle, Academic Search Complete y PsycInfo no ofrecían resultados ajustados a lo esperado, por lo que se decidió eliminarlas del estudio. La búsqueda en las cuatro bases restantes proporcionó un total de 679478 registros (Gráfico 3). Después de ajustar la búsqueda con base en los filtros expuestos en el paso 3 los regis- tros se redujeron a 2230. De estos registros, 2189 fueron descartados al no cumplir con los criterios de inclusión. Una vez realizada la selección se revisaron los textos completos de los 41 estudios restantes; de ellos, 29 cumplían los criterios de inclusión; tras revisar las referencias de los artículos seleccionados se decidió añadir 4 trabajos más al estudio, ya que cumplían dichos criterios.

Gráfico 3. Procedimiento de la búsqueda sistemática.

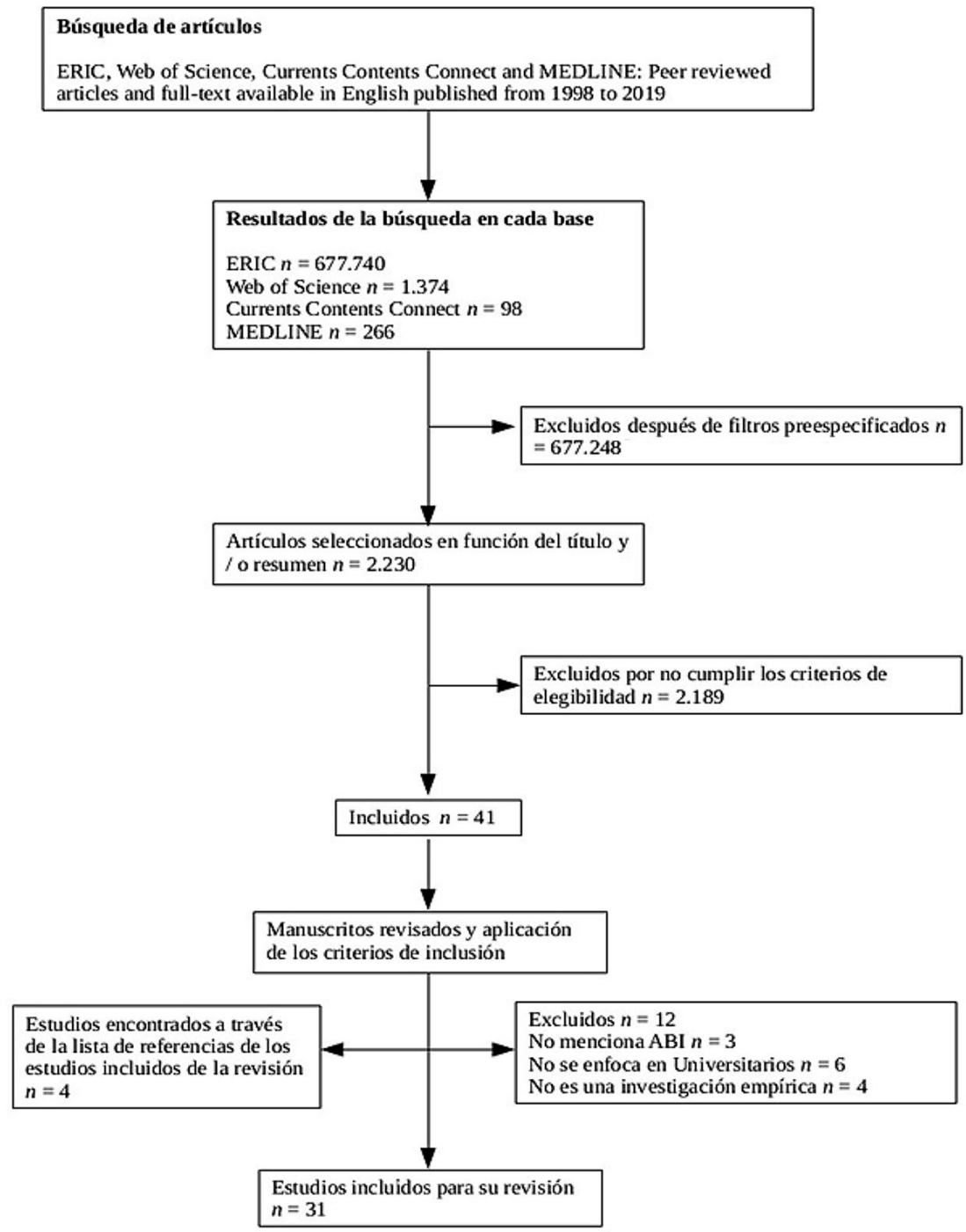

Fuente: Elaboración propia. 
Finalmente, en la revisión sistemática se analizaron 31 artículos que describían la aplicación del ABI en titulaciones de ciencias sociales y de la salud.

El análisis de cada uno de los artículos seleccionados se realizó a partir de las siguientes preguntas:

1. ¿Cuál es la procedencia, duración y características metodológicas de los estudios?

2. ¿Cuál es la modalidad de ABI que utilizan?

3. ¿Qué tipo de objetivos se plantean y cuáles son los efectos en el alumnado?

4. ¿Qué limitaciones tienen los estudios analizados?

\section{Resultados y discusión}

3.1. Duración, procedencia y características metodológicas de los estudios

El $16 \%$ de los estudios analizados se realizó a lo largo de un semestre, el $22 \%$ durante un año académico, un $25 \%$ se realizó en algunas sesiones 0 meses, un $12 \%$ durante varios años y un $25 \%$ no indicaba la duración de la intervención. Respecto al país de realización de los estudios, el $29 \%$ fueron llevados a cabo en Inglaterra, el $19 \%$ en EEUU, el $12 \%$ en Australia, el $9 \%$ en España, otro $9 \%$ en Turquía, y el $22 \%$ en China, Tailandia, Canadá, Irán, Nueva Zelanda, Indonesia y Arabia Saudí, países donde solo se realiza un estudio. En cuanto a las principales características metodológicas de los estudios, el $36 \%$ empleaba una metodología cuantitativa, el $48 \%$ eran estudios cualitativos y el $16 \%$ utilizaba una metodología mixta (Tabla 1).

a) Estudios cuantitativos. El $84 \%$ son experimentales, el $8 \%$ cuasi-experimentales y el $8 \%$ descriptivos. Los estudios de corte cuantitativo suelen ser: 1) experimentales, con grupo control y grupo experimental, con un análisis pretest-postest por medio de cuestionarios estandarizados, en un tiempo limitado de experimentación (Irwanto, Saputro y Prodjosantoso, 2018; Konokman y Yelken, 2016; Piyayodilokchai, Panjaburee, Laosinchai, Ketpichainarong y Ruenwongsa, 2013); 2) longitudinales, en los que se evalúa el progreso de los estudiantes, comenzando con una metodología convencional e introduciendo paulatinamente la metodología ABI (Zafra-Gómez, Román-Martínez y Gómez-Miranda, 2015). Kienzler y Fontanesi (2017) plantean un estudio por pasos en el que: 1) se crean pequeños grupos de alumnos que formulan un desafío de investigación bien estructurado relacionado con el KTE (Knowledge, Translation and Exchange [conocimiento, traducción e intercambio]) con la finalidad de romper la brecha del «conocimiento a la acción»; 2) los alumnos presentan sus desafíos al grupo de trabajo para discutirlo de manera crítica; 3 ) eligen los desafíos para seguir investigando; 4) se plantea un taller para discutir los desafíos; 5) se evalúa el proceso de aprendizaje mediante un formulario de retroalimentación a mitad y al final del curso.

b) Estudios cualitativos. El $50 \%$ eran estudios descriptivos, el $22 \%$ eran estudios de caso único, el $14 \%$ etnografías y el $14 \%$ era de tipo interpretativo. 


\begin{tabular}{|c|c|c|c|c|c|c|c|c|c|c|c|c|c|c|}
\hline & 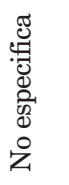 & 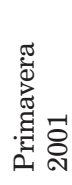 & 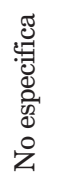 & 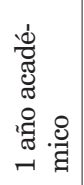 & 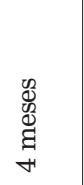 & 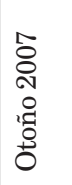 & 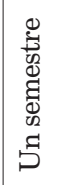 & 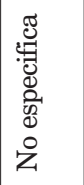 & 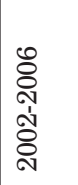 & 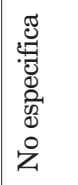 & 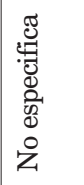 & 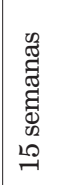 & 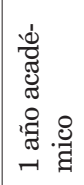 & 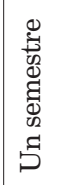 \\
\hline D. & 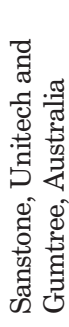 & 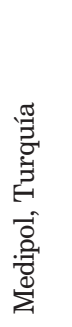 & 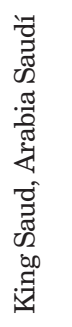 & 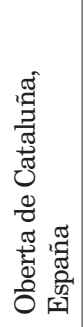 & 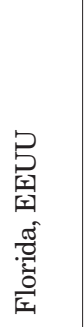 & 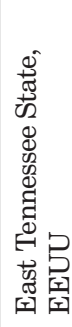 & 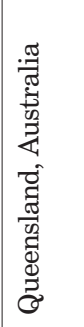 & 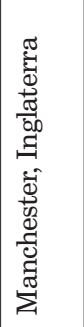 & 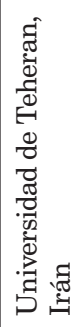 & 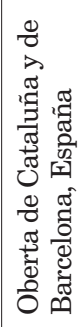 & 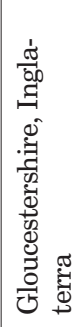 & 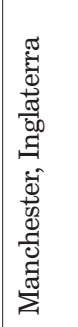 & 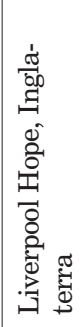 & 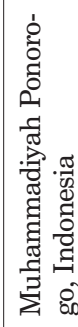 \\
\hline$\frac{\pi}{2}$ & 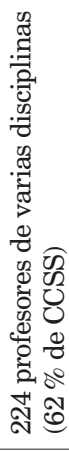 & 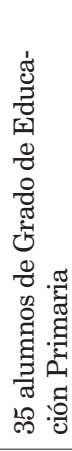 & 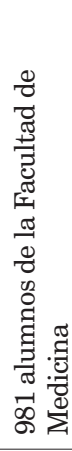 & 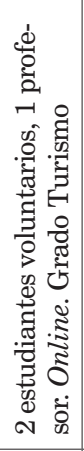 & 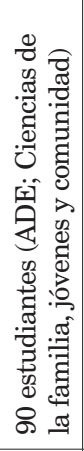 & 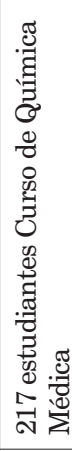 & 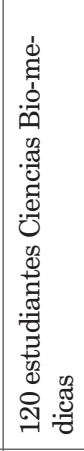 & 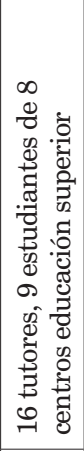 & 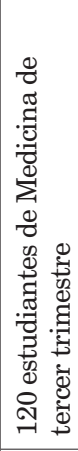 & 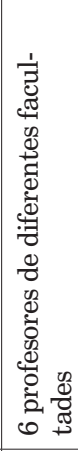 & 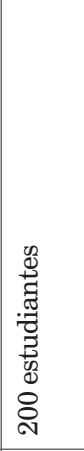 & 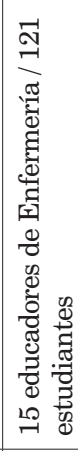 & 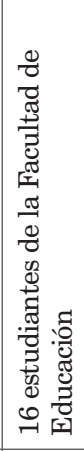 & 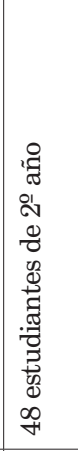 \\
\hline & 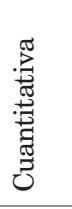 & 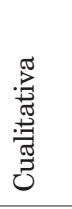 & 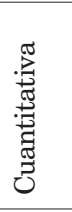 & 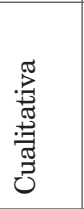 & 焉 & 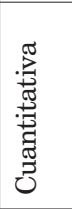 & 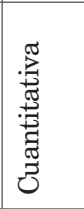 & 䠞 & 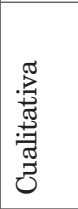 & 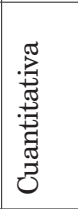 & 蕞 & 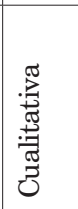 & 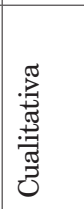 & 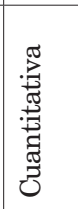 \\
\hline & 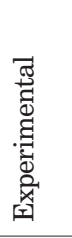 & 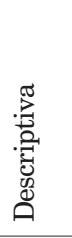 & 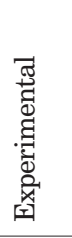 & 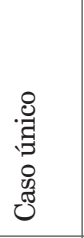 & 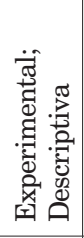 & 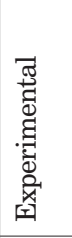 & 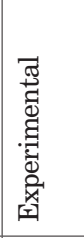 & 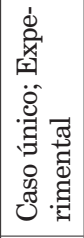 & 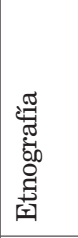 & 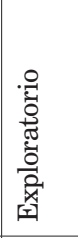 & 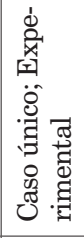 & 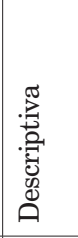 & 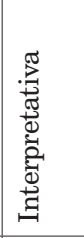 & 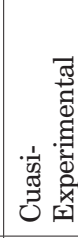 \\
\hline $\begin{array}{l}0 \\
\vdots \\
0\end{array}$ & 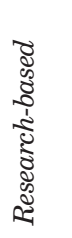 & 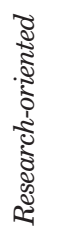 & 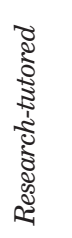 & 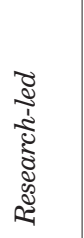 & 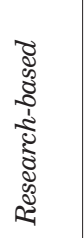 & 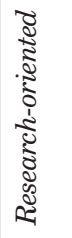 & 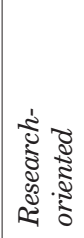 & 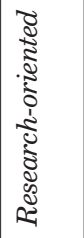 & 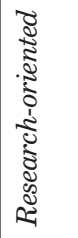 & 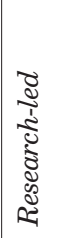 & 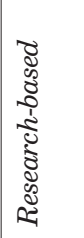 & 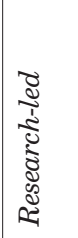 & 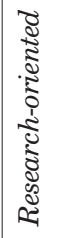 & 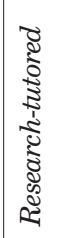 \\
\hline & 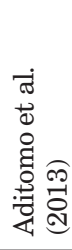 & 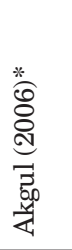 & 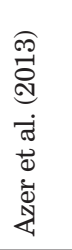 & 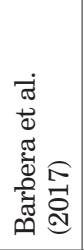 & 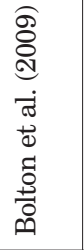 & 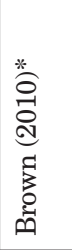 & 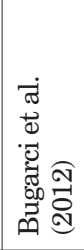 & 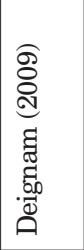 & 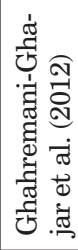 & 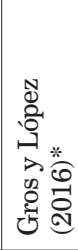 & 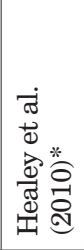 & 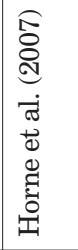 & 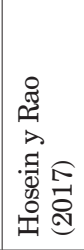 & 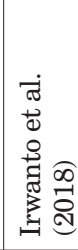 \\
\hline & & & & & & & $\Sigma$ & $\infty$ & $\sigma$ & - & $\exists$ & $\stackrel{\mathcal{N}}{\stackrel{1}{*}}$ & $\stackrel{\infty}{\rightarrow}$ & $\exists$ \\
\hline
\end{tabular}




\begin{tabular}{|c|c|c|c|c|c|c|c|c|c|c|c|c|c|c|c|c|}
\hline 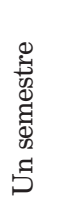 & 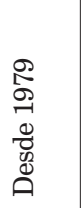 & 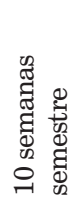 & 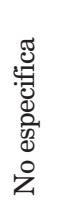 & 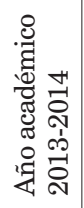 & 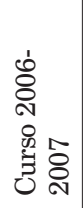 & 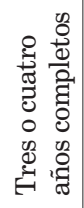 & 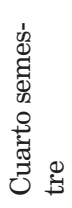 & 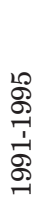 & 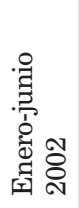 & 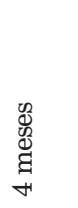 & 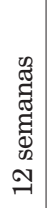 & 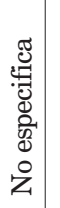 & 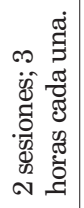 & 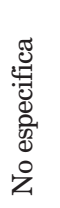 & 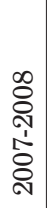 & 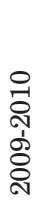 \\
\hline 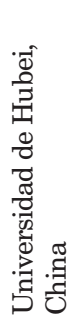 & 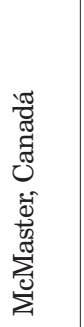 & 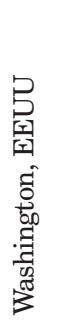 & 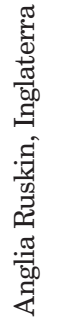 & 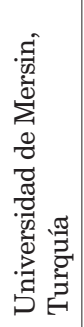 & 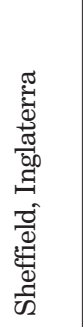 & 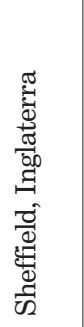 & 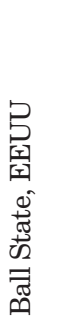 & 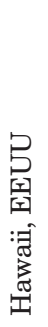 & 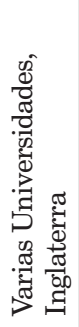 & 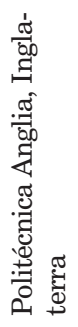 & 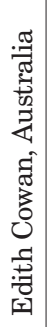 & 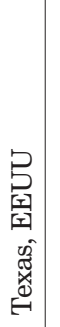 & 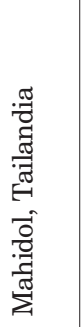 & 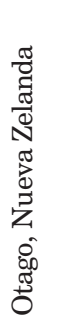 & 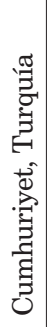 & 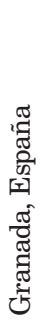 \\
\hline 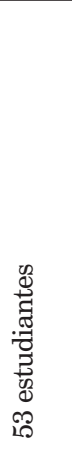 & 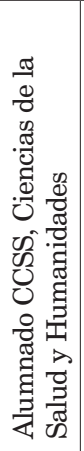 & 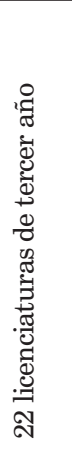 & 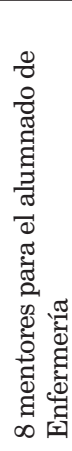 & 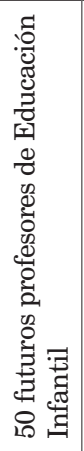 & 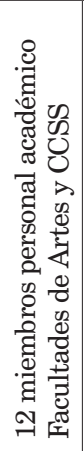 & 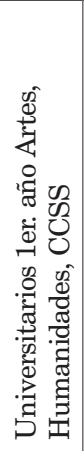 & 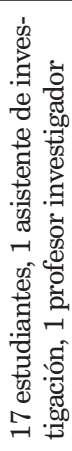 & 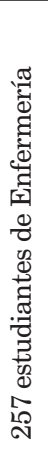 & 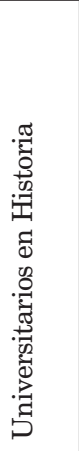 & 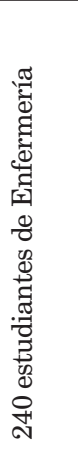 & 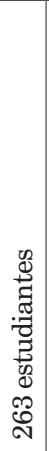 & 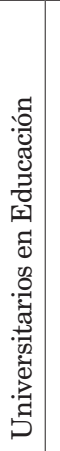 & 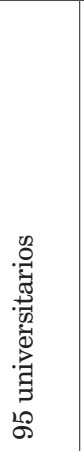 & 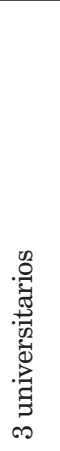 & 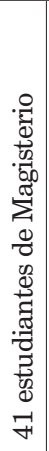 & 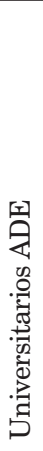 \\
\hline 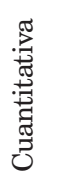 & 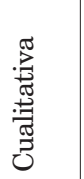 & 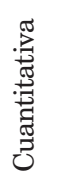 & 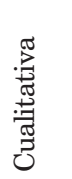 & $\frac{\pi}{x^{\frac{\pi}{2}}}$ & 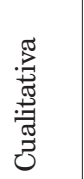 & 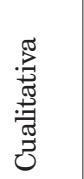 & 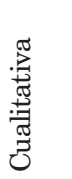 & 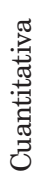 & 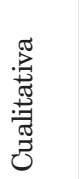 & 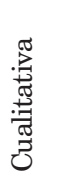 & 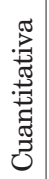 & 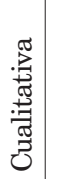 & 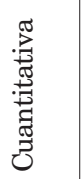 & 离 & 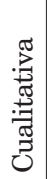 & 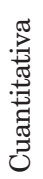 \\
\hline
\end{tabular}

\begin{tabular}{|c|c|c|c|c|c|c|c|c|c|c|c|c|c|c|c|c|}
\hline & 0 & 0 & 0 & 乙 & 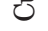 & 0 & 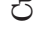 & 0 & 0 & 0 & 0 & 0 & 0 & z & 0 & 0 \\
\hline 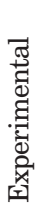 & 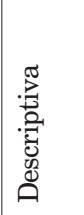 & 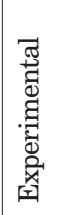 & 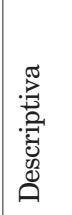 & 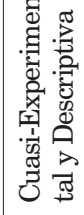 & 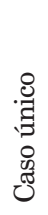 & 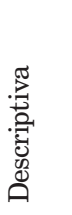 & 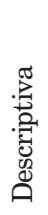 & 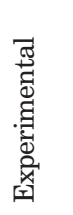 & 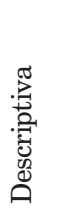 & 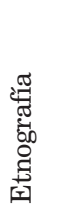 & 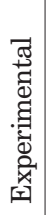 & 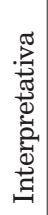 & 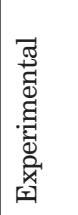 & 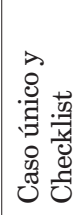 & 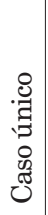 & 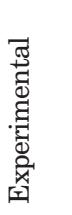 \\
\hline 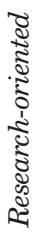 & 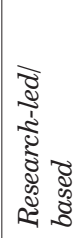 & 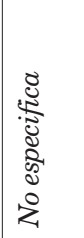 & 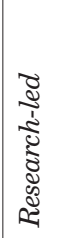 & 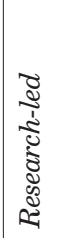 & 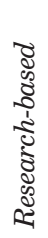 & 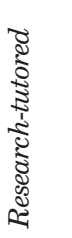 & 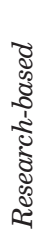 & 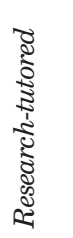 & 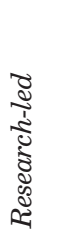 & 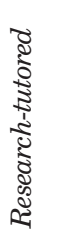 & 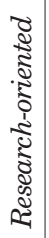 & 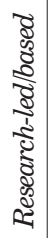 & 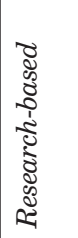 & 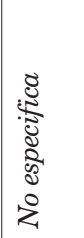 & 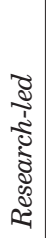 & 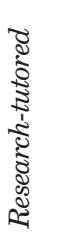 \\
\hline 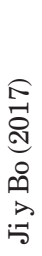 & 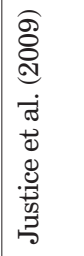 & 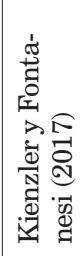 & 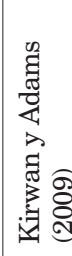 & 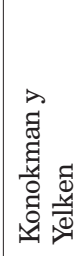 & 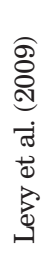 & 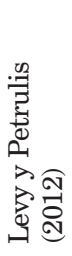 & 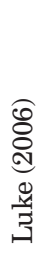 & 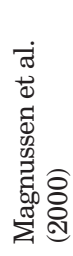 & 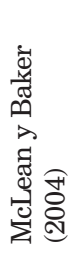 & 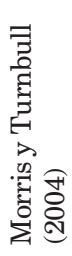 & 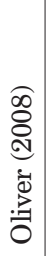 & 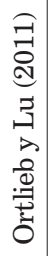 & 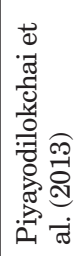 & 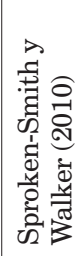 & 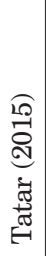 & 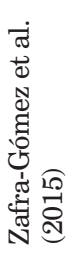 \\
\hline$\stackrel{20}{-1}$ & $\stackrel{\bullet}{\sim}$ & 듬 & $\stackrel{\infty}{\sim}$ & 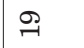 & 오 & $\vec{N}$ & Nิ & ลึ & ন゙ & $\stackrel{10}{N}$ & $\stackrel{\sim}{*}$ & ล & $\stackrel{\infty}{\sim}$ & ని & คి & $\vec{m}$ \\
\hline
\end{tabular}

$\stackrel{\varrho}{\mathrm{J}_{2}}$ \% כ N $\frac{\mathbb{D}}{\mathrm{D}}$ 
Los estudios cualitativos tienen como principal característica el tiempo de dedicación al programa 0 proceso de enseñanza (Barbera, García y Fuertes-Alpiste, 2017; Ghahremani-Ghajar, Mohammadi Doostdar y Sadegh Mirhosseini, 2012; Levy y Petrulis, 2012; Tatar, 2015). Por ejemplo, la finalidad del estudio de Justice et al. (2009) era indagar sobre la introducción de la metodología ABI en la Universidad McMaster (Canadá) desde el año 1979; sus informantes eran el profesorado, administradores e instructores, a quienes se entrevistaba para conocer de primera mano la experiencia de adaptar el ABI. Barbera et al. (2017) y Levy et al. (2009) realizaron estudios de caso único, centrados en la información obtenida de personas clave a través de entrevistas. Hosein y Rao (2017), Levy y Petrulis (2012), McLean y Barker (2004) y Ortlieb y Lu (2011) llevaron a cabo estudios descriptivos e interpretativos en los que se analizaban ensayos de reflexión y entrevistas con el programa ATLAS.ti.

c) Metodologías mixtas. El $20 \%$ combinan un método experimental e interpretativo; el $40 \%$ un estudio de caso único y un estudio experimental; el $20 \%$ un estudio cuasi-experimental y un estudio interpretativo; y el $20 \%$ un estudio de caso único y una checklist de extracción de datos cuantitativos. En el estudio realizado por Spronken-Smith y Walker (2010) se les aplicó a tres profesores que utilizaban la metodología ABI una checklist sobre el enfoque de la investigación, se les observó durante la sesiones de clase y se les entrevistó para indagar sobre los procesos y los resultados obtenidos.

\subsection{Modalidad de ABI}

Los estudios muestran las cuatro modalidades del ABI descritas por Griffiths (2004) y Healey (2005) (ver Tabla 1). El $29 \%$ de los estudios analizados utilizaban la modalidad Research-led, enfatizando la elaboración de un proceso de construcción del conocimiento dominado por los intereses de la propia institución; el $26 \%$ de los estudios desarrollan la modalidad Research-oriented, centrando el proceso de aprendizaje del alumnado en la investigación y en la manera de crear el conocimiento; el $26 \%$ de los estudios se basaba en la modalidad Research-based, pues el proceso de enseñanza se centraba en el papel activo del estudiante en dicho proceso, minimizando el rol del profesorado; y el $19 \%$ en la modalidad Research-tutored, ya que el proceso de enseñanza se centra en pequeños grupos de discusión guiados por el profesorado, el cual les ofrecía retroalimentación de los progresos realizados.

Tras la identificación de las modalidades en cada uno de los estudios analizados, surgió la duda de si todos conceptualizaban de la misma manera la metodología ABI. Muchos autores entienden la metodología ABI como una forma de enseñanza constructivista que conecta al estudiante con el aprendizaje, permitiéndole explorar desde diferentes perspectivas la investigación y la creación del conocimiento (Healey, Jordan, Pell y Short, 2010; Levy y Petrulis, 2012; Spronken-Smith y Walker, 2010; Zafra-Gómez et al., 2015).

Aunque varían los términos utilizados para describir las cuatro modalida- 
des del ABI, hay coincidencia al describir la participación del alumnado de una forma inductiva, fomentando la asunción de la responsabilidad hacia su propio aprendizaje y el intercambio de conocimientos al trabajar en grupos. Existen autores que identifican la metodología ABI indistintamente con la metodología de aprendizaje basado en problemas; ambas se consideran parte de una orientación filosófica común en la consecución de un aprendizaje inductivo (Azer, Hasanato, Al-Nassar, Somily y AlSaadi, 2013; Deignan, 2009; Horne et al., 2007; Kirwan y Adams, 2009; Morris y Turnbull, 2004).

Según Ghahremani-Ghajar et al. (2012) el ABI parte de un enfoque amplio de enseñanza-aprendizaje, al surgir de una variedad de interpretaciones y prácticas enraizadas en el aprendizaje basado en problemas $(\mathrm{ABP})$, que originalmente se proponía en la educación médica. Aditomo et al. (2013) señalan que el ABI se sustenta en enfoques pedagógicos orientados a la consecución de un aprendizaje basado en la investigación; en este caso, su aplicación se realiza mediante estrategias de aprendizaje basado en problemas, aprendizaje basado en proyectos y aprendizaje basado en casos. Aunque existen diferencias en cuanto a la manera de conceptualizar el ABI, uniéndolo con otros enfoques pedagógicos o no, todos los autores identifican su metodología como una oportunidad para conseguir un aprendizaje inductivo, ya que: a) permite la asunción de la responsabilidad en el aprendizaje y en la contribución activa en el proceso de enseñanza; b) ofrece multitud de beneficios en la formación de futuros profesionales de la educación y la sanidad (Hosein y Rao, 2017; Ji y Bo, 2017; Magnussen, Ishida y Itano, 2000; Oliver, 2008).

\subsection{Objetivos de los estudios y efectos de la aplicación del $A B I$ en el alumnado}

En los estudios analizados se identificaron cinco objetivos en la aplicación del $\mathrm{ABI}$, así como los efectos que esta metodología tenía en el alumnado universitario (Tabla 2).

Objetivo 1. Examinar el efecto de aplicar la metodología $A B I$ en el proceso de enseñanza-aprendizaje. El $33 \%$ de los estudios pretendían evaluar cuáles eran los principales efectos de aplicar la metodología ABI. Por ejemplo, analizar las resistencias del alumnado a la aplicación del ABI para la preparación de historias digitales, o el uso de medios alternativos partiendo del modelo de inferencia triádico de Pierce (Konokman y Yelken, 2016; Ortlieb y Lu, 2011); observar cómo asumían los estudiantes la información sobre la situación económica y financiera actual tras haber trabajado el tema (Zafra-Gómez et al., 2015); examinar posibles resistencias de los departamentos para la aplicación del $\mathrm{ABI}$ y los beneficios que implica para los egresados (Justice et al., 2009). Los estudios evidenciaron: 1) efectos positivos tras la aplicación de la metodología ABI, siempre y cuando el proceso estuviese estructurado de manera adecuada; 2) un aumento significativo de los conocimientos y habilidades del alumnado a corto y largo plazo; 3) una mejora de su rendimiento académico (Azer et al., 2013; Konokman y Yelken, 2016; Justice et al., 2009). 
Lidia E. SANTANA-VEGA, Arminda SUÁREZ-PERDOMO y Luis FELICIANO-GARCÍA

TABLA 2. Efectos de la aplicación del ABI en el alumnado.

\begin{tabular}{|c|c|c|c|c|c|c|c|c|}
\hline & Autores y año & $\begin{array}{l}\text { Aumento } \\
\text { del cono- } \\
\text { cimiento }\end{array}$ & $\begin{array}{l}\text { Habili- } \\
\text { dades de } \\
\text { búsqueda }\end{array}$ & $\begin{array}{l}\text { Rendi- } \\
\text { miento } \\
\text { académico }\end{array}$ & $\begin{array}{c}\text { Auto- } \\
\text { confianza }\end{array}$ & $\begin{array}{l}\text { Auto- } \\
\text { aprendi- } \\
\text { zaje }\end{array}$ & Motivación & $\begin{array}{l}\text { Pensa- } \\
\text { miento } \\
\text { crítico }\end{array}$ \\
\hline 1 & Aditomo et al. (2013) & Sí & Sí & - & - & Sí & Sí & Sí \\
\hline 2 & Akgul (2006) & Sí & Sí & - & - & Sí & - & - \\
\hline 3 & Azer et al. (2013) & Sí & Sí & Sí & Sí & Sí & Sí & - \\
\hline 4 & Barbera et al. (2017) & Sí & - & - & - & Sí & Sí & - \\
\hline 5 & Bolton et al. (2009) & Sí & - & - & - & Sí & Sí & Sí \\
\hline 6 & Brown (2010) & Sí & Sí & - & Sí & - & - & Sí \\
\hline 7 & Bugarci et al. (2012) & Sí & Sí & - & - & Sí & - & - \\
\hline 8 & Deignam (2009) & Sí & Sí & Sí & - & Sí & Sí & - \\
\hline 9 & $\begin{array}{l}\text { Ghahremani-Ghajar, et } \\
\text { al. (2012) }\end{array}$ & Sí & - & - & - & Sí & - & Sí \\
\hline 10 & Gros y López (2016) & Sí & - & - & - & Sí & - & - \\
\hline 11 & Healey et al. (2010) & Sí & Sí & - & - & Sí & Sí & - \\
\hline 12 & Horne et al. (2007) & Sí & Sí & - & Sí & - & Sí & Sí \\
\hline 13 & Hosein y Rao (2017) & Sí & Sí & - & Sí & Sí & Sí & - \\
\hline 14 & Irwanto et al. (2018) & Sí & Sí & Sí & - & Sí & - & Sí \\
\hline 15 & Ji y Bo (2017) & Sí & Sí & - & - & Sí & Sí & - \\
\hline 16 & Justice et al. (2009) & Sí & - & Sí & - & Sí & Sí & - \\
\hline 17 & $\begin{array}{l}\text { Kienzler y FontaneSí } \\
\text { (2017) }\end{array}$ & Sí & Sí & - & Sí & Sí & Sí & Sí \\
\hline 18 & Kirwan y Adams(2009) & Sí & - & - & Sí & Sí & Sí & - \\
\hline 19 & $\begin{array}{l}\text { Konokman y Yelken } \\
(2016)\end{array}$ & Sí & Sí & Sí & - & Sí & Sí & - \\
\hline 20 & Levy et al. (2009) & Sí & Sí & - & - & Sí & Sí & - \\
\hline 21 & Levy y Petrulis (2012) & Sí & - & Sí & Sí & Sí & Sí & Sí \\
\hline 22 & Luke (2006) & Sí & - & Sí & - & Sí & - & - \\
\hline 23 & Magnussen et al. (2000) & Sí & - & - & - & - & Sí & Sí \\
\hline 24 & McLean y Baker (2004) & Sí & - & - & - & Sí & Sí & Sí \\
\hline 25 & $\begin{array}{l}\text { Morris y Turnbull } \\
\text { (2004) }\end{array}$ & Sí & - & - & Sí & Sí & Sí & - \\
\hline 26 & Oliver (2008) & Sí & Sí & - & Sí & Sí & Sí & - \\
\hline 27 & Ortlieb y Lu (2011) & Sí & Sí & - & Sí & Sí & Sí & Sí \\
\hline 28 & $\begin{array}{l}\text { Piyayodilokchai et al. } \\
\text { (2013) }\end{array}$ & Sí & Sí & Sí & - & Sí & Sí & - \\
\hline 29 & $\begin{array}{l}\text { Sproken-Smith y } \\
\text { Walker (2010) }\end{array}$ & Sí & Sí & - & - & Sí & Sí & - \\
\hline 30 & Tatar (2015) & Sí & Sí & - & - & Sí & Sí & - \\
\hline 31 & $\begin{array}{l}\text { Zafra-Gómez et al. } \\
\text { (2015) }\end{array}$ & Sí & - & Sí & Sí & Sí & Sí & Sí \\
\hline
\end{tabular}

Fuente: Elaboración propia. 
Los resultados del estudio realizado por Levy et al. (2009) mostraron el gran potencial de la metodología $\mathrm{ABI}$, al ofrecer oportunidades de reflexión y discusión que fomentan un alto grado de empoderamiento; para ello, el profesorado necesita tener acceso a una amplia gama de experiencias de ABI, algunas estructuradas y otras dirigidas por los estudiantes.

El alumnado indicó la necesidad de tener en cuenta el aspecto social del ABI, ya que aprendían del esfuerzo de sus compañeros, además del suyo propio; aunque eran conscientes del posible aumento de ansiedad y estrés en el proceso de aprendizaje, lo percibían de manera positiva por los beneficios para su aprendizaje (Deignan, 2009; Ji y Bo, 2017; Luke, 2006). Healey et al. (2010) observaron que el ABI fomenta el interés del alumnado por el contenido curricular y aumenta su motivación al pensar que es factible cursar estudios de posgrado.

Objetivo 2. Evaluar el desarrollo de competencias de investigación en los estudiantes. El $11 \%$ de los estudios pretendía examinar la conciencia de los propios alumnos sobre el desarrollo de competencias investigadoras en la elaboración de ensayos de reflexión (Hosein y Rao, 2017); evaluar cómo se comprende y experimenta el proceso de enseñanza basada en la indagación, relacionándolo con la construcción epistemológica del alumnado (Levy y Petrulis, 2012). Estos estudios evidenciaron un creciente entusiasmo del alumnado en el aprendizaje de la investigación, entendiendo el potencial de una metodología centrada en el estudiante para su formación futura (Hosein y Rao, 2017; Levy y Petrulis, 2012). Akgul (2006) observó que los estudiantes percibían la ciencia como un proceso para encontrar más verdades o hechos, y que sus niveles de aprendizaje dependían de su implicación y compromiso.

Objetivo 3. Promover tareas o resultados de aprendizaje por la aplicación del ABI. El $22 \%$ de los estudios utilizan el ABI como una forma de introducir valores relacionados con el voluntariado en una organización sin ánimo de lucro (Bolton, Brennan y Terry, 2009), o de investigar el logro del alumnado cuando se asume un modelo de ciclo de aprendizaje complementado con recursos multimedia (Piyayodilokchai et al., 2013). Se observó que la metodología es efectiva para revertir el rol del estudiante como un mero receptor pasivo; los estudiantes pudieron aprender cómo se gestiona el aprendizaje, asumían desafíos e incrementaban su autoconfianza (Bolton et al., 2009; Kienzler y Fontanesi, 2017). Aditomo et al. (2013) identificaron, tras aplicar el ABI, ocho tareas de investigación para la consecución de resultados de aprendizaje satisfactorios: investigación académica, investigación simplificada, literatura basada en la indagación, discusión basada en la indagación, investigación aplicada, investigación aplicada simulada, promulgación de la práctica y role playing. Tras la aplicación del ABI se lograba una amplia gama de objetivos educativos que involucraban aspectos cognitivos, metacognitivos, afectivos, sociales y epistemológicos.

Objetivo 4. Explorar el desarrollo del pensamiento crítico. El $19 \%$ de los estudios 
pretendía explorar cuáles eran los puntos de vista críticos del alumnado cuando se plantea un desafío de investigación en el aprendizaje idiomático (GhahremaniGhajar et al., 2012), o examinar los cambios en el pensamiento crítico de los estudiantes después de la aplicación del ABI, comparando su opinión al inicio de la carrera con la opinión al final de la misma (Magnussen et al., 2000; Tatar, 2015). Se puso de manifiesto que si la metodología $\mathrm{ABI}$ estaba bien estructurada y se realizaba en pequeños grupos, aportaba beneficios al alumnado universitario; se observó, a su vez, un gran impacto en el pensamiento crítico y en las habilidades de resolución de problemas (Irwanto et al., 2018; Ghahremani-Ghajar et al., 2012; Tatar, 2015; Gros y López, 2016). Bugarcic, Zimbardi, Macaranas y Thorn, (2012) observaron que el uso de la metodología ABI promueve un aprendizaje significativo y estimula a los estudiantes a plantear ideas y evaluar críticamente aquello que se encuentre en un entorno de investigación «real», a la vez que obtienen un alto nivel de conocimiento detallado del contenido.

Objetivo 5. Promover habilidades y competencias personales para el autoaprendizaje. El $15 \%$ de los estudios tenían la finalidad de examinar si la aplicación del ABI tiene impacto en la motivación, la autoconfianza, el autoaprendizaje, las actitudes científicas, el compromiso de participación (Ji y Bo, 2017; Oliver, 2008; Brown, 2016), o examinar la capacidad del alumnado para asumir autonomía en el aprendizaje, de manera positiva y/o negativa, y sus reacciones e interpretaciones ante la investigación cíclica (Luke, 2006). Los estudiantes reporta- ban que la metodología ABI estimulaba el deseo por el conocimiento, despertaba el entusiasmo por el autoaprendizaje y fortalecía la autoeficacia y la motivación.

En general, los estudios señalan: a) un aumento de la motivación del alumnado, b) una mejora en la comprensión de las materias y de su relevancia para la sociedad, c) un incremento de la colaboración entre el alumnado al trabajar conjuntamente para conseguir un objetivo común, d) un aumento en la corresponsabilidad ante la consecución de la tarea, e) una mejora de las habilidades interpersonales y del desempeño de roles de trabajo (Bruder y Prescott, 2013; Frezell, 2018). El $100 \%$ de los estudios constató que la aplicación del ABI favorecía el incremento del conocimiento en el alumnado; el $61 \%$ evidenció un desarrollo de las habilidades investigadoras; el $29 \%$ mostró un aumento del rendimiento académico; el $36 \%$ un aumento de la autoconfianza en los estudiantes; el $90 \%$ un aumento en el autoaprendizaje del alumnado; el $70 \%$ una mayor motivación hacia el aprendizaje; y el $40 \%$ un aumento del pensamiento crítico.

\subsection{Algunas limitaciones de los estudios}

En este apartado se presentan algunas limitaciones de los estudios:

1) Las investigaciones se han llevado a cabo a partir de un determinado modelo de ABI y con grupos muy específicos de estudiantes; si bien se obtiene una instantánea de la experiencia de los estudiantes y de los aspectos facilitadores en un determinado contexto, se necesita explorar la aplicación 
del ABI con otras muestras para considerar la generalización de sus resultados (Horne et al., 2007; Spronken-Smith y Walker, 2010).

2) En algunos estudios las limitaciones provienen de las estructuras universitarias, ya que se pueden producir resistencias en los departamentos al no encontrar beneficios obvios en la implementación del ABI, o al considerar que podría alterar el flujo de poder y la asignación de los recursos existentes (Justice, Rice, Roy, Hudspith y Jenkins, 2009).

3) Otras limitaciones provienen del método de investigación utilizado; al usar un método cualitativo se asume el riesgo de sesgar las respuestas de los estudiantes hacia un enfoque excesivamente optimista del ABI, si las entrevistas las realiza el investigador principal (Luke, 2006). El uso de grupos focales puede influir en los resultados ya que, en ocasiones, hablan más los estudiantes que disfrutaron de la clase 0 interactuaron con el profesorinvestigador (Luke, 2006; Morris y Turnbull, 2004). Con respecto a la metodología cuantitativa, el uso de instrumentos de medidas estandarizados, al ofrecer solo la respuesta cerrada del alumnado, soslaya las vivencias, experiencias y emociones generadas en el proceso (Magnussen et al., 2000).

\section{Conclusiones}

En la revisión sistemática se identificaron 31 artículos sobre experiencias de $\mathrm{ABI}$ en ciencias sociales y de la salud. Estas experiencias no se circunscriben a un solo país y abordan la aplicación del ABI desde diferentes perspectivas de investigación (cuantitativa, cualitativa y mixta). Los objetivos marcados en los estudios no solo se centran en averiguar la posibilidad de aplicar el ABI en el aula, sino también en entender cuáles son los principales efectos en el proceso de enseñanza, qué competencias de investigación adquieren los estudiantes, cómo se promueven resultados de aprendizaje, cómo se desarrolla el pensamiento crítico, y cómo se fomentan habilidades y competencias personales. Para descubrir si esta metodología puede ser beneficiosa para el proceso de enseñanza-aprendizaje y el desarrollo de competencias investigadoras en el alumnado, los autores aplicaron diferentes modalidades de ABI y determinaron sus fortalezas y debilidades. Como fortalezas los autores señalan: la exploración con mayor grado de profundidad de los conocimientos, la promoción del aprendizaje cooperativo, el compromiso del alumnado en su autoaprendizaje y el aumento de un pensamiento crítico. Entre las debilidades se señalan: la incapacidad de cubrir las expectativas de aprendizaje del alumnado y las reticencias de las estructuras universitarias ante el $\mathrm{ABI}$.

El ABI favorece el aprendizaje significativo de los estudiantes universitarios, al involucrarlos en un proceso de elaboración de una investigación, y fortalece el nexo indagación-enseñanza, siempre y cuando el proceso permita a los actores expresar sus vivencias y emociones. Para llevar a cabo experiencias de ABI se ha de enfatizar en el alumnado la necesidad de construir el conocimiento y potenciar 
su responsabilidad en el proceso de enseñanza-aprendizaje (Healey, 2005; Levy y Petrulis, 2012). La metodología del ABI proporciona un alto andamiaje social y un aprendizaje activo, fomentando habilidades personales para la investigación, la corresponsabilidad en la consecución de las tareas y la capacidad de reflexión en situaciones de aprendizaje (Bruder y Prescott, 2013; Frezell, 2018; Hmelo-Silver et al., 2007).

\section{En definitiva, el ABI propicia un proce-} so de enseñanza que: a) fomenta el desarrollo de competencias de investigación de una cohorte estudiantil que asume el desafío de su autoaprendizaje; b) proporciona un espacio de creación de conocimiento estimulado por la indagación; y c) fomenta el interés y el compromiso de los estudiantes por su proceso de aprendizaje, y por elaborar trabajos con calidad académica.

Las principales limitaciones del presente estudio radican en el hecho de restringir la búsqueda: 1) a los artículos sobre ABI escritos en lengua inglesa, y 2) a los artículos que aplicaban esta metodología en las titulaciones pertenecientes al ámbito de las ciencias sociales y de la salud en el contexto universitario. Es necesario que en próximas investigaciones se amplíen los criterios de búsqueda, incorporando los trabajos realizados en español, que abarquen otras áreas de conocimiento y otras etapas educativas. No obstante, nuestro estudio aporta información valiosa para identificar las posibilidades de aplicar el $\mathrm{ABI}$ en titulaciones que forman a futuros profesionales del campo de las ciencias sociales y de la salud.

\section{Referencias bibliográficas}

Aditomo, A., Goodyear, P., Bliuc, A. M. y Ellis, R. A. (2013). Inquiry-based learning in higher education: principal forms, educational objectives, and disciplinary variations. Studies in Higher Education, 38 (9), 1239-1258. doi: https://doi.or $\mathrm{g} / 10.1080 / 03075079.2011 .616584$

Åkerlind, G. S. (2008). An academic perspective on research and being a researcher: an integration of the literature. Studies in Higher Education, 33 (1), 17-31. doi: https://doi. org $/ 10.1080 / 03075070701794775$

Akgul, E. M. (2006). Teaching science in an inquiry-based learning environment: What it means for pre-service elementary science teachers. Eurasia Journal of Mathematics, Science and Technology Education, 2 (1), 71-81. doi: https://doi.org/10.12973/ejmste/75439

Azer, S. A., Hasanato, R., Al-Nassar, S., Somily, A. y AlSaadi, M. M. (2013). Introducing integrated laboratory classes in a PBL curriculum: impact on student's learning and satisfaction. $B M C$ Medical Education, 13 (1), 71. doi: https://doi. org/10.1186/1472-6920-13-71

Barbera, E., Garcia, I y Fuertes-Alpiste, M. (2017). A Co-Design Process Microanalysis: Stages and Facilitators of an Inquiry-Based and Technology-Enhanced Learning Scenario. The International Review of Research in Open and Distributed Learning, 18 (6), 105-125. doi: https://doi.org/10.19173/irrodl.v18i6.2805

Bevins, S. y Price, G. (2016). Reconceptualising inquiry in science education. International Journal of Science Education, 38 (1), 17-29. doi: https://doi.org/10.1080/09500693.2015.1124300

Bolton, E. B., Brennan, M. A. y Terry, B. D. (2009). Students Learn How Nonprofits Utilize Volunteers through Inquiry-Based Learning. International Journal of Teaching and Learning in Higher Education, 21 (3), 285-294.

Brew, A. (2003). Teaching and research: New relationships and their implications for inquiry-based teaching and learning in higher education. Higher Education Research \& Development, 22 (1), 3-18. doi: https://doi. org/10.1080/0729436032000056571

Brown, J. A. (2016). Evaluating the effectiveness of a practical inquiry based learning bioinformatics module on undergraduate student en- 
gagement and applied skills. Biochemistry and molecular biology education, 44 (3), 304-313. doi: https://doi.org/10.1002/bmb.20954

Bruder, R. y Prescott, A. (2013). Research evidence on the benefits of IBL. ZDM Mathematics Education, 45 (6), 811-822. doi: https://doi. org/10.1007/s11858-013-0542-2

Bugarcic, A., Zimbardi, K., Macaranas, J. y Thorn, P. (2012). An inquiry based practical for a large, foundation level undergraduate laboratory that enhances student understanding of basic cellular concepts and scientific experimental design. Biochemistry and Molecular Biology Education, 40 (3), 174-180. doi: https:/doi. org/10.1002/bmb.20587

Deignan, T. (2009). Enquiry-Based Learning: Perspectives on practice. Teaching in Higher Education, 14 (1), 13-28. doi: https://doi. org/10.1080/13562510802602467

Frezell, D. (2018). Impact of Inquiry Based Learning on Students' Motivation, Engagement and Attitude in Science. Electronic Theses and Dissertations, 7356. Recuperado de https://scholar.uwindsor.ca/etd/7356 (Consultado el 18-11-2019).

Ghahremani-Ghajar, S. S., Mohammadi Doostdar, H. y Sadegh Mirhosseini, S. M. (2012). We have been living with this pain: Enquiry-based language learning in Iranian higher education. Teaching in Higher Education, 17 (3), 269-281. doi: https://doi.org/10.1080/13562517.2011.611 864

Griffiths, R. (2004). Knowledge production and the research-teaching nexus: The case of the built environment disciplines. Studies in Higher Education, 29 (6), 709-726. doi: https://doi. org $/ 10.1080 / 0307507042000287212$

Gros, B. y López, M. (2016). Students as co-creators of technology-rich learning activities in higher education. International Journal of Educational Technology in Higher Education, 13 (1), 28. doi: https://doi.org/10.1186/s41239-016-0026-x

Healey, M. (2005). Linking research and teaching exploring disciplinary spaces and the role of inquiry-based learning. En R. Barnett (Coord.), Reshaping the university: New relationships between research, scholarship and teaching (pp. 67-78). Buckingham: Open University Press.
Healey, M. y Jenkins., A. (2009). Developing undergraduate research and inquiry. Research Report to the Higher Education Academy. York: Higher Education Academy.

Healey, M., Jordan, F., Pell, B. y Short, C. (2010). The research-teaching nexus: a case study of students' awareness, experiences and perceptions of research. Innovations in Education and Teaching International, 47 (2), 235-246. doi: https://doi.org/10.1080/14703291003718968

Herman, W. E. y Pinard, M. R. (2015). Critically Examining inquiry-based learning: John Dewey in theory, history, and practice. In Inquiry-Based Learning For Multidisciplinary Programs: A Conceptual and Practical Resource for Educators (pp. 43-62). Bingley, Yorkshire: Emerald Group Publishing Limited. Hmelo-Silver, C. E., Duncan, R. G. y Chinn, C. A. (2007). Scaffolding and achievement in problem-based and inquiry learning: a response to Kirschner, Sweller, and Clark. Educational Psychologist, 42 (2), 99-107. doi: https://doi. org/10.1080/00461520701263368

Horne, M., Woodhead, K., Morgan, L., Smithies, L., Megson, D. y Lyte, G. (2007). Using enquiry in learning: From vision to reality in higher education. Nurse Education Today, 27 (2), 103-112. doi: https://doi.org/10.1016/j.nedt.2006.03.004

Hosein, A. y Rao, N. (2017). Students' reflective essays as insights into student centred-pedagogies within the undergraduate research methods curriculum. Teaching in Higher Education, 22 (1), 109-125. doi: https://doi.org/10.1080/135 62517.2016.1221804

Hunter, A. B., Laursen, S. L. y Seymour, E. (2007). Becoming a scientist: The role of undergraduate research in students' cognitive, personal, and professional development. Science Education, 91 (1), 36-74. doi: https://doi.org/10.1002/ sce. 20173

Irwanto, S., Saputro, A. D. y Prodjosantoso, A. K. (2018). Promoting Critical Thinking and Problem Solving Skills of Preservice Elementary Teachers through Process-Oriented GuidedInquiry Learning (POGIL). International Journal of Instruction, 11 (4), 777-794. doi: https:// doi.org/10.12973/iji.2018.11449a

Ji, F. y Bo, H. (2017). Function Design for Inquiry-Based Learning Platform Based on a 
Computer Network. International Journal of Emerging Technologies in Learning (iJET), 12 (02), 40-51. doi: https://doi.org/10.3991/ijet. v12i02.6043

Justice, C., Rice, J., Roy, D., Hudspith, B. y Jenkins, H. (2009). Inquiry-based learning in higher education: administrators' perspectives on integrating inquiry pedagogy into the curriculum. Higher Education, 58 (6), 841. doi: https:// doi.org/10.1007/s10734-009-9228-7

Justice, C., Rice, J. y Warry, W. (2009). Developing Useful and Transferable Skills: Course Design to Prepare Students for a Life of Learning. International Journal for the scholarship of Teaching and Learning, 3 (2), 1-19. doi: https://doi. org/10.20429/ijsotl.2009.030209

Justice, C., Rice, J., Warry, W., Inglis, S., Miller, S. y Sammon, S. (2007). Inquiry in higher education: Reflections and directions on course design and teaching methods. Innovative Higher Education, 31 (4), 201-214. doi: https://doi. org/10.1007/s10755-006-9021-9

Kahn, P. y O'Rourke, K. (2004). Guide to curriculum design: Enquiry-based learning. Recuperado de https://www.academia.edu/460509/ Guide_to_Curriculum_Design_Enquiry-Based_Learning (Consultado el 26-11-2019).

Kienzler, H. y Fontanesi, C. (2017). Learning through inquiry: A global health hackathon. Teaching in Higher Education, 22 (2), 129-142. doi: https://doi.org/10.1080/13562517.2016.122 1805

Kirwan, A. y Adams, J. (2009). Students' views of enquiry-based learning in a continuing professional development module. Nurse Education Today, 29 (4), 448-455. doi: https://doi. org/10.1016/j.nedt.2008.09.003

Konokman, Y. y Yelken, T. (2016). Preparing Digital Stories through the Inquiry-Based Learning Approach: Its Effect on Prospective Teachers' Resistive Behaviors toward Research and Technology-Based Instruction. Educational Sciences: Theory and Practice, 16 (6), 2141-2165. doi: https://doi.org/10.12738/estp.2016.6.0410

Levy, P., Aiyegbayo, O. y Little, S. (2009). Designing for inquiry based learning with the Learning Activity Management System. Journal of Computer Assisted Learning, 25 (3), 238-251. doi: https://doi.org/10.1111/j.1365-2729.2008.00309.x
Levy, P. y Petrulis, R. (2012). How do first-year university students experience inquiry and research, and what are the implications for the practice of inquiry-based learning? Studies in Higher Education, 37 (1), 85-101. doi: https:// doi.org/10.1080/03075079.2010.499166

Liberati, A., Altman, D. G., Tetzlaff, J., Mulrow, C., Gøtzsche, P. C., Ioannidis, J. P., .... y Moher, D. (2009). The PRISMA statement for reporting systematic reviews and meta-analyses of studies that evaluate health care interventions: explanation and elaboration. PLoS medicine, 6 (7), e1000100. doi: https://doi.org/10.1371/ journal.pmed. 1000100

Luke, C. L. (2006). Fostering Learner Autonomy in a Technology Enhanced, Inquiry Based Foreign Language Classroom. Foreign Language Annals, 39 (1), 71-86. doi: https://doi. org/10.1111/j.1944-9720.2006.tb02250.x

Maass, K. y Engeln, K. (2018). Effects of Scaled-up Professional Development Courses About Inquiry-Based Learning on Teachers. Journal of Education and Training Studies, 6 (4), 1-16. doi: https://doi.org/10.11114/jets.v6i4.3083

Magnussen, L., Ishida, D. y Itano, J. (2000). The impact of the use of inquiry-based learning as a teaching methodology on the development of critical thinking. Journal of Nursing Education, 39 (8), 360-364. doi: https://doi. org/10.3928/0148-4834-20001101-07

McLean, M. y Barker, H. (2004). Students making progress and the 'research teaching nexus' debate. Teaching in Higher Education, 9 (4), 407-419. doi: https://doi. org $/ 10.1080 / 1356251042000252354$

Minner, D. D., Levy, A. J. y Century, J. (2010). Inquiry based science instruction-what is it and does it matter? Results from a research synthesis years 1984 to 2002. Journal of Research in Science Teaching: The Official Journal of the National Association for Research in Science Teaching, 47 (4), 474-496. doi: https:// doi.org/10.1002/tea.20347

Morris, D. y Turnbull, P. (2004). Using student nurses as teachers in inquiry-based learning. Journal of Advanced Nursing, 45 (2), 136-144. doi: https://doi.org/10.1046/j.13652648.2003.02875.x 
Oliver, R. (2008). Engaging first year students using a web-supported inquiry-based learning setting. Higher Education, 55 (3), 285-301. doi: https://doi.org/10.1007/s10734-007-9055-7

Ortlieb, E. y Lu, L. (2011). Improving teacher education through inquiry-based learning. International Education Studies, 4 (3), 41-46. doi: https://doi.org/10.5539/ies.v4n3p41

Piyayodilokchai, H., Panjaburee, P., Laosinchai, P., Ketpichainarong, W. y Ruenwongsa, P. (2013). A 5E Learning Cycle Approach-Based, Multimedia-Supplemented Instructional Unit for Structured Query Language. Journal of Educational Technology \& Society, 16 (4), 146-159.

Prince, M. y Felder, R. (2007). The many faces of inductive teaching and learning. Journal of College Science Teaching, 36 (5), 14.

Spronken-Smith, R., Angelo, T., Matthews, H., O'Steen, B. y Robertson, J. (2007). How effective is inquiry-based learning in linking teaching and research. En An International Colloquium on International Policies and Practices for Academic Enquiry (pp. 19-21). Winchester, UK: Marwell.

Spronken-Smith, R. y Walker, R. (2010). Can inquiry based learning strengthen the links between teaching and disciplinary research? Studies in Higher Education, 35 (6), 723-740. doi: https://doi.org/10.1080/03075070903315502

Tatar, N. (2015). Pre-service teachers' beliefs about the image of a science teacher and science teaching. Journal of Baltic Science Education, 14 (1), 34-44.

Zafra-Gómez, J. L., Román-Martínez, I. y GómezMiranda, M. E. (2015). Measuring the impact of inquiry-based learning on outcomes and student satisfaction. Assessment \& Evaluation in Higher Education, 40 (8), 1050-1069. doi: https://doi.org/10.1080/02602938.2014.963836

\section{Biografía de los autores}

Lidia E. Santana-Vega es Doctora en Filosofía y Ciencias de le Educación, y Licenciada en Psicología. Catedrática de
Universidad adscrita al Departamento de Didáctica e Investigación Educativa de la Facultad de Educación de la Universidad de La Laguna. Coordinadora del grupo de investigación consolidado GIOES. Sus líneas de investigación se centran en la atención/apoyo al alumnado, los proyectos de vida de jóvenes en riesgo de exclusión social y la toma de decisiones y género.

iD http://orcid.org/0000-0002-2543-6543

Arminda Suárez-Perdomo es Doctora en Psicología Evolutiva y Profesora del Departamento de Familia, Escuela y Sociedad de la Universidad Internacional de la Rioja (UNIR). Sus líneas de investigación se centran en el fomento de la parentalidad positiva en entornos virtuales de aprendizaje experiencial y en la competencia digital de padres y madres, así como en la influencia de la procrastinación en el rendimiento académico del alumnado universitario.

\section{0} https://orcid.org/0000-0002-6755-5284

Luis Feliciano-García es Doctor en Filosofía y Ciencias de la Educación, y Profesor Titular del Departamento de Didáctica e Investigación Educativa en la Facultad de Educación de la Universidad de La Laguna. Miembro del Grupo de Investigación GIOES. Su línea de investigación se centra en las metodologías activas y en la toma de decisiones académico-laborales. http://orcid.org/0000-0002-2909-4990 


\section{revista española de pedagogía año 78, n. ${ }^{\circ}$ 277, septiembre-diciembre 2020}

Spanish Journal of Pedagogy

year 78, n. 277, September-December 2020

\section{Sumario*}

\section{Table of Contents **}

\section{Dominio del tiempo y desarrollo personal y social Mastering time and personal and social development}

Editoras invitadas: Ana Ponce de León Elizondo y M. ${ }^{2}$ Ángeles Valdemoros San Emeterio

Guest editors: Ana Ponce de León Elizondo, \& M. 'ángeles Valdemoros San Emeterio

Ana Ponce de León Elizondo y M. Ángeles Valdemoros San Emeterio

Presentación: Dominio del tiempo y desarrollo personal y social

Introduction: Mastering time and personal and social development

Antonio Bernal Guerrero, M. Ángeles Valdemoros San Emeterio y Alfredo Jiménez Eguizábal Tiempo, poder y educación. Repensando la construcción de la identidad personal y las decisiones de la política educativa

Time, power, and education. Rethinking the construction of personal identity and educational policy decisions

José Antonio Caride

Educar y educarnos a tiempo, pedagógica y socialmente To educate and educate ourselves in time, pedagogically and socially
Rosa Ana Alonso Ruiz, Magdalena Sáenz de Jubera Ocón y Eva Sanz Arazuri

Tiempos compartidos entre abuelos y nietos, tiempos de desarrollo personal

Shared time between grandparents and grandchildren: A time for personal development 415

Nuria Codina, Rafael Valenzuela y José Vicente Pestana De la percepción a los usos del tiempo: perspectiva temporal y procrastinación de adultos en España From the perception to the uses of time: Time perspective and procrastination among adults in Spain

José Manuel Muñoz-Rodríguez, Patricia Torrijos Fincias, Sara Serrate González y Alicia Murciano Hueso Entornos digitales, conectividad y educación.

Percepción y gestión del tiempo en la construcción de la identidad digital de la juventud

Digital environments, connectivity and education: Time perception and management in the construction of young people's digital identity

Ángel De-Juanas Oliva, Francisco Javier GarciaCastilla y Ana Ponce de León Elizondo El tiempo de los jóvenes en dificultad social: utilización, gestión y acciones socioeducativas The time of young people in social difficulties: Use, management, and socio-educational actions

\footnotetext{
* Todos los artículos están también publicados en inglés en la página web de la revista: https://revistadepedagogia.org.

** All the articles are also published in English on the web page of the journal: https://revistadepedagogia.org.
} 


\section{Estudios}

Studies

Catherine L'Ecuyer y José Ignacio Murillo

El enfoque teleoóógico de la educación Montessori

y sus implicaciones

Montessori's teleological approach to education and its

implications

\section{Lidia E. Santana-Vega, Arminda Suárez-Perdomo y Luis Feliciano-García}

El aprendizaje basado en la investigación en el contexto universitario: una revisión sistemática Inquiry-based learning in the university context:

A systematic review

519

\section{Reseñas bibliográficas}

Moreno, A. (2020). Personalizar, un modelo para una educación de calidad en el siglo xxl. Informe Delphi de

499
Expertos (Cristina Medrano Pascual). Fuentes, J. L. (Coord.) (2019). Ética para la excelencia educativa (Ana García.Bravo).

\section{Informaciones}

Congreso Virtual Educa: «World Congress. Human

capital development for social innovation. Connecting the Americas, Africa and Europen; 8th International Congress of Educational Sciences and Development; Cambios en los congresos anunciados en números anteriores a raíz de la pandemia por el Covid.19.

Índice del año 2020

Table of contents of the year 2020 551 Instrucciones para los autores Instructions for authors 559

ISSN: 0034-9461 (Impreso), 2174-0909 (Online)

https://revistadepedagogia.org/

Depósito legal: M. 6.020 - 1958

INDUSTRIA GRÁFICA ANZOS, S.L. Fuenlabrada - Madrid 


\section{Inquiry-based learning in the university context: A systematic review*}

\section{El aprendizaje basado en la investigación en el contexto universitario: una revisión sistemática}

Lidia E. SANTANA-VEGA, PhD. Professor. Universidad de La Laguna (Isantana@ull.es).

Arminda SUÁREZ-PERDOMO, PhD. Assistant Professor. Universidad de La Laguna (asuper@ull.edu.es).

Luis FELICIANO-GARCÍA, PhD. Associate Professor. Universidad de La Laguna (Ifelici@ull.edu.es).

\section{Abstract:}

Inquiry-Based Learning (IBL) is a methodology that enhances learning through a knowledge construction process. The aim of this study is to establish how IBL is used and what effects it has on university students from social sciences and health sciences. This study follows the PRISMA guidelines for conducting systematic reviews. It comprises an analysis of 31 studies extracted from four electronic databases and reference lists on the topic, published in English between 19982019. The results show that the strengths of IBL are: 1) promoting cooperative learning,
2) engaging students in self-learning, and 3) increasing critical thinking. Its weaknesses include: 1) the inability to meet learning expectations, and 2) the reluctance of university hierarchies to embrace IBL. The potential of IBL as a teaching strategy at university level is discussed as it allows deep knowledge construction, increased learning motivation, and development of students' research skills as well as their self-learning, self-confidence, critical thinking, and academic performance. IBL favours meaningful learning by university students by offering a space for the creation of knowledge stimulated by the inquiry process.

\footnotetext{
* This work is part of a larger project funded by the European Commission (Education, Audiovisual and Culture Executive Agency, EACEA), REFLECT LAB: Supporting lecturers in applying inquiry based learning (2016-DE01-KA203-002891). Erasmus + Strategic Partnerships in the field of higher education. This publication reflects the views only of the authors, and the Commission cannot be held responsible for any use which may be made of the information contained therein.
}

Revision accepted: 2020-06-22.

This is the English version of an article originally printed in Spanish in issue 277 of the revista española de pedagogía. For this reason, the abbreviation EV has been added to the page numbers. Please, cite this article as follows: SantanaVega, L. E., Suárez-Perdomo, A., Feliciano-García, L. (2020). El aprendizaje basado en la investigación en el contexto universitario: una revisión sistemática Inquiry-based learning in the university context: A systematic review. Revista Española de Pedagogía, 78 (277), 519-537. doi: https://doi.org/10.22550/REP78-3-2020-08 
Keywords: inquiry-based learning, college students, teaching methods, active learning, systematic review, PRISMA.

\section{Resumen:}

$\mathrm{El}$ aprendizaje basado en la investigación (ABI) es una metodología que potencia los aprendizajes mediante un proceso de construcción del conocimiento. La finalidad del estudio era conocer cómo se está aplicando el ABI y sus efectos en estudiantes universitarios de ciencias sociales y de la salud. El método para realizar la revisión sistemática ha seguido las directrices de la declaración PRISMA. Se analizaron un total de 31 estudios extraídos de cuatro bases de datos electrónicas y listas de referencias sobre el tópico, publicados en inglés entre 1998-2019. Los resultados mostraron como fortalezas del ABI: 1) la promoción del aprendizaje cooperativo; 2) el compromiso del alumnado en su autoaprendizaje, y 3) el aumento de un pensamiento crítico. Entre sus debilidades se señalan:1) la incapacidad de cubrir las expectativas de aprendizaje, y 2) las reticencias de las estructuras universitarias hacia el ABI. A partir de estos resultados se discute el valor del ABI como estrategia didáctica en la universidad, en la medida en que permite profundizar en la construcción del conocimiento, incrementar la motivación de aprendizaje, desarrollar las habilidades de investigación, el autoaprendizaje, la autoconfianza, el pensamiento crítico, y el rendimiento académico. El $\mathrm{ABI}$ favorece el aprendizaje significativo en el alumnado universitario al ofrecer un espacio de creación de conocimiento estimulado por el proceso de indagación.

Descriptores: aprendizaje basado en la investigación, alumnado universitario, métodos de enseñanza, aprendizaje activo, revisión sistemática, PRISMA.

\section{Introduction}

Inquiry-based learning (IBL) encompasses a variety of teaching focuses deriving from the perspectives of Dewey and Bruner (Herman \& Pinard, 2015) who argue that inquiry is at the centre of tasks, the use of resources, and learning instructions. IBL sets students challenges that: a) catalyse their engagement and participation, b) promote experiential learning, and c) stimulate exploration and the search for solutions (Aditomo, Goodyear, Bliuc, \& Ellis, 2013; Levy, Aiyegbayo, \& Little, 2009; Oliver, 2008; Prince \& Felder, 2007; Spronken-Smith,
Angelo, Matthews, O'Steen, \& Robertson 2007). IBL is a promising approach for improving teaching and learning processes in universities.

IBL promotes a pedagogy centred on the student as an agent who actively seeks out and constructs knowledge (Healey \& Jenkins, 2009; Justice, Rice, \& Warry, 2009; Sproken-Smith \& Walker, 2010). The importance of inquiry-based pedagogies resides in fostering students' capacity to adopt learning strategies that use inquiry techniques and tools, allowing them to construct their own knowledge 
in greater depth (Levy \& Petrulis, 2012). In IBL: a) scientific enquiry is built into the teaching-learning process; b) teaching focusses on the learner; c) learning is stimulated by inquiry when questions or doubts arise; d) teaching staff play a role as knowledge facilitators; e) learning is the outcome of a knowledge construction process that fosters cognition and metacognition; f) self-directed learning is stimulated (Aditomo et al., 2013; Levy \& Petrulis, 2012; Spronken-Smith \& Walker, 2010; Justice et al., 2007; Kahn \& O'Rourke, 2004).

IBL provides students with a broad social scaffolding and guidance for managing their inquiry (Hmelo-Silver, Duncan, \& Chinn, 2007); it promotes active learning with positive effects on students' achievements and their attitudes towards research (Maass \& Engeln, 2018); it stimulates their problem-solving capacity, critical thinking, and reflection on learning (Bruder \& Prescott, 2013; Minner, Levy, \& Century, 2010); it fosters university students' research competence and training, improving the quality of what they learn and the process of peer collaboration (Bevins \& Price, 2016); it promotes better understanding of subjects among students, as they accept the challenges their academic training involves (Akkerlind, 2008; Brew, 2003; Healey \& Jenkins, 2009; Hunter, Laursen, \& Seymour, 2007); and it increases their academic writing skills (Justice, Rice, \& Warry, 2009).

Griffiths (2004) and Healey (2005) identify four IBL modes depending on how the link between teaching and research is built:

a) Research-led. The curriculum is dominated by the interests of teaching staff who set the information transmission model. Students learn about the results of research.

b) Research-oriented. Teachers attempt to create a research ethic through teaching; the curriculum emphasises knowledge-production processes and the learning achieved. Students learn through the research process.

c) Research-based. The division between the roles of student and teacher is minimised. The curriculum is largely designed around inquiry-based activities. Students learn as researchers.

d) Research-tutored. Students learn on the basis of the results of research, formulated in small discussion groups with a teacher who facilitates the process.

According to Healey (2005), the curriculum must be redesigned to make the research-teaching nexus practical, planning a teaching process centred on students as the authors of their own learning. IBL has mainly been implemented in science subjects such as maths, physics, and biology. Systematic review studies of the application of IBL with university students from social and/or healthcare courses are rare. In order to establish how IBL is applied in these courses, we performed a study following the 
guidelines from the PRISMA statement for performing systematic reviews and meta-analyses (Liberati et al., 2009). The objectives of this study are:

1. To identify the duration, source, and methodological characteristics of the studies analysed.

2. To examine the type of IBL mode used.

3. To analyse the objectives of the studies on the implementation of IBL and its effects on social science and health science students.

4. To analyse the limitations of the studies.

\section{Method}

\subsection{Search and eligibility criteria}

To search for publications about the use of IBL with university students from the social sciences and health sciences, we proposed a series of inclusion and exclusion criteria: a) articles must specifically state the use of active methodologies such as IBL; articles that only mention "Research Teaching Nexus", "Learning Strategies", "Compe-
tence-Based Learning" are excluded; b) the study population must be university students; studies focussing on non-university students are rejected; c) the date range of the publications included in the review must be 1998-2019; articles published before then are excluded; d) the selected publications must be limited to the fields of social sciences (education, psychology, anthropology, social work, etc.) and health sciences (medicine, nursing, physiotherapy, etc.); articles focussing on qualifications from other areas of knowledge are excluded; e) the articles must be written in English; those written in other languages are excluded.

The systematic review was carried out using various online databases: ERIC, Web of Science, Current Contents Connect, MEDLINE, PsycArticles, Academic Search Complete, and PsycInfo. These databases were selected for their relevance as they contain scientific articles published in indexed journals. To identify the search terms relating to the topic to be studied, we performed a preliminary literature search and consulted experts in IBL. We performed an iterative search in each database, combining the two sets of terms shown in Graph 1.

\section{GRAPH 1. Truncated search strategy.}

$$
\begin{aligned}
& \text { su(Inquiry-based learning OR Enquiry-based learning OR Guided- } \\
& \text { inquiry* OR Inquiry-based learning cycle OR Research teaching nexus } \\
& \text { OR research-based* method) AND su(method of teaching IBL* OR } \\
& \text { active learning OR Undergraduates* research OR self-directed learning } \\
& \text { OR student-centred OR student-focus OR High education) }
\end{aligned}
$$

Source: Own elaboration. 
We used thesaurus terms (Graph 2) in the databases that offer this search option to find entries with the exact terms, according to the inclusion criteria established in the study.

GRAPH 2. Thesaurus terms search strategy.

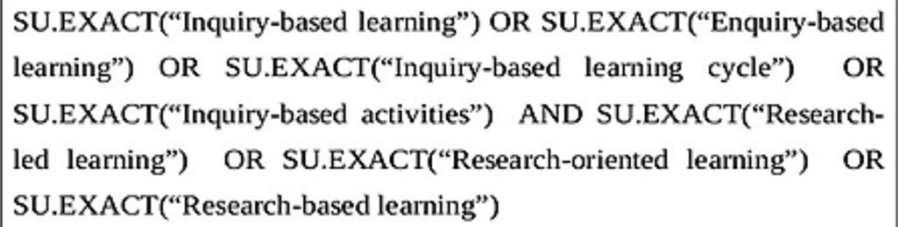

Source: Own elaboration.

\subsection{Data collection and analysis proce- dure}

The steps in this search were:

1. Establishing truncated search terms and thesaurus terms to limit the searches in accordance with the objectives of the study.

2. Searching for entries in the selected databases.

3. Ordering the entries obtained by relevance. When the number of search results exceeded 100 entries, we used the following filters to reduce the number: works subjected to double blind review; full texts with links; works from the 1998-2019 period; works published in English in academic journals.

4. Carrying out a second selection of the entries retrieved, using the title and/or abstract as the criteria and excluding ones that do not match the thematic area.

5. Making a data extraction sheet for each article with the inclusion criteria specifying: the reference of the study; the reason for selection; title and/or abstract (university population, social sciences or health sciences, IBL); year of publication. Finally, their suitability was determined for subsequent analysis.

6. Verifying the degree of accuracy of the data extracted in the selection of articles.

After a preliminary search in each database, we found that PsycArticle, Academic Search Complete, and PsycInfo did not return results that fitted what we wanted, and so we decided to eliminate them from the study. The search of the four remaining databases gave a total of 679,478 entries (Graph 3). After adjusting the search on the basis of the filters described in step 3, the number of entries was reduced to 2,230. Of these entries, 2,189 were rejected as they did not match the inclusion criteria. Once we had carried out the selection, we reviewed the complete texts of the 41 remaining studies. Of these, 29 met the inclusion cri- 
teria. After reviewing the references in the selected articles, we decided to add 4 more works to the study as they fulfilled these criteria. Finally, in the systematic review we analysed 31 articles describing the application of IBL in social science and health science courses.
Each of the selected articles was analysed based on the following questions:

1. What are the origin, duration, and methodological characteristics of the studies?

2. What IBL mode do they use?

\section{GrapH 3. Systematic Search Procedure.}

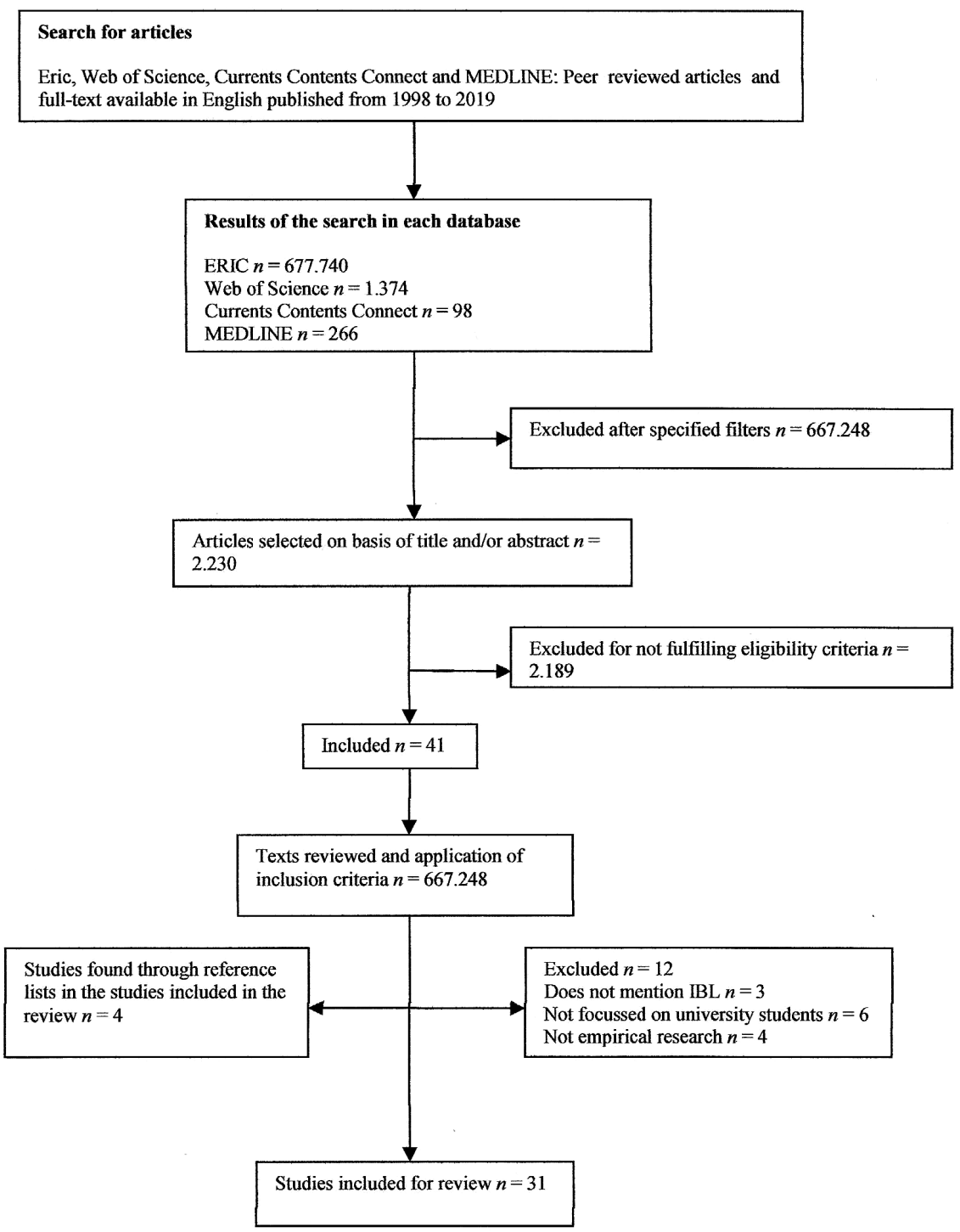

Source: Own elaboration. 
3. What type of objectives are set and what are the effects on the students?

4. What are the limitations of the studies analysed?

\section{Results and discussion}

\subsection{Duration, origin, and methodologi- cal characteristics of the studies}

Of the studies analysed, 16\% were carried out over one semester, $22 \%$ over an academic year, $25 \%$ in a few sessions or months, $12 \%$ over several years, and $25 \%$ did not state the duration of the intervention. Regarding the country where the studies were performed, $29 \%$ were carried out in England, 19\% in the USA, 12\% in Australia, 9\% in Spain, another 9\% in Turkey, and the remaining 22\% in China, Thailand, Canada, Iran, New Zealand, Indonesia, and Saudi Arabia, with only one study from each of these countries. Regarding the main methodological characteristics of the studies, 36\% used a quantitative methodology, $48 \%$ were qualitative studies, and $16 \%$ used a mixed methodology (Table 1).

a) Quantitative studies. Of these, $84 \%$ are experimental, 8\% quasi-experimental, and $8 \%$ descriptive. The quantitative studies are generally: 1) experimental, with a control group and an experimental group, using pretest-posttest analysis with standardised questionnaires in a limited experimentation time (Irwanto, Saputro, \& Prodjosantoso, 2018; Konokman \& Yelken, 2016; Piyayodilokchai, Panjaburee, Laosinchai, Ketpichainarong, \& Ruenwongsa, 2013); 2) longitudinal, evaluating students' progress, starting with a conventional methodology and gradually introducing the IBL methodology (Zafra-Gómez, Román-Martínez, \& Gómez-Miranda, 2015). Kienzler and Fontanesi (2017) propose a step-by-step study in which: 1) small groups of students were created who formulated a well-structured research challenge relating to KTE (Knowledge, Translation and Exchange) with the aim of breaching the "knowledge to action" barrier; 2) the students presented their challenges to the working groups for critical discussion; 3) they chose the challenges to continue to investigate; 4) the challenges were discussed in a workshop; 5) the learning process was evaluated using a feedback form halfway through the course and at the end of it.

b) Qualitative studies. Of these, 50\% were descriptive, $22 \%$ were single-case studies, $14 \%$ ethnographic, and $14 \%$ were interpretative. The main characteristic of the qualitative studies was the time dedicated to the teaching programme or process (Barbera, García, \& Fuertes-Alpiste 2017; Ghahremani-Ghajar, Mohammadi Doostdar, \& Sadegh Mirhosseini, 2012; Levy \& Petrulis, 2012; Tatar, 2015). For example, the aim of the study by Justice et al. (2009) was to investigate the use of IBL at McMaster University (Canada) since 1979; the informants were the teaching staff, administrative staff, and instructors who were interviewed to establish first-hand the experience of adapting IBL. Barbera et al. (2017) and Levy et al. (2009) carried out single-case studies, focussed on the information obtained from key respondents through interviews. 


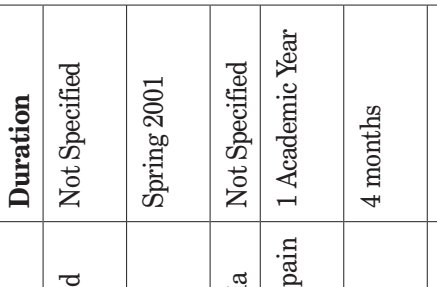

\begin{tabular}{|c|c|c|c|c|c|c|c|c|c|c|c|c|c|c|c|c|}
\hline 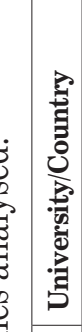 & 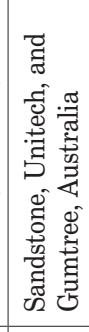 & 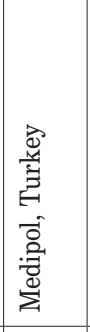 & 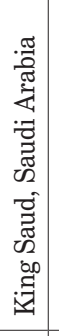 & 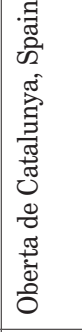 & 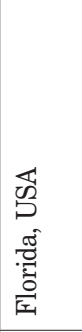 & 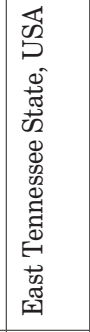 & 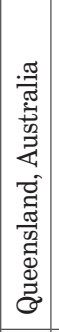 & 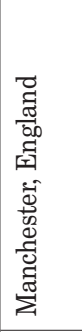 & 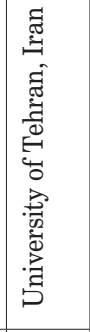 & 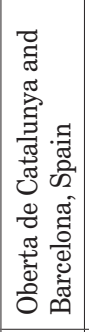 & 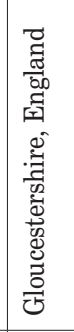 & 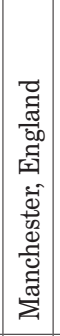 & 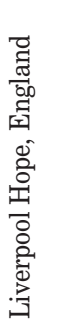 & 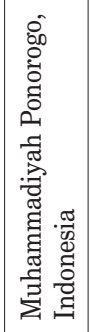 & 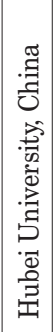 & 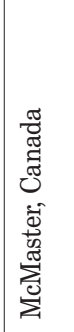 \\
\hline 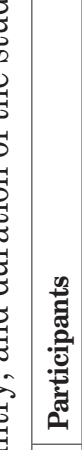 & 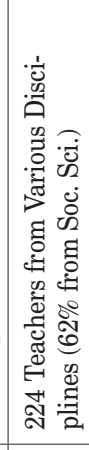 & 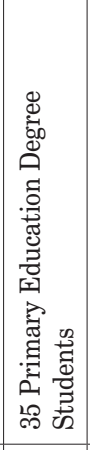 & 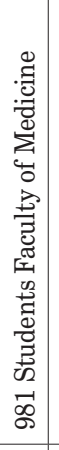 & 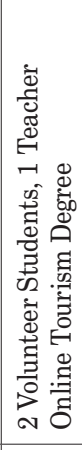 & 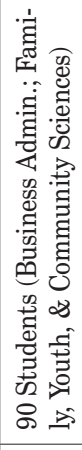 & 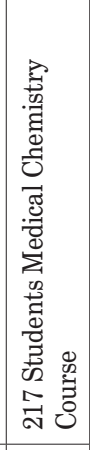 & 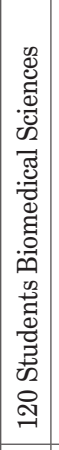 & 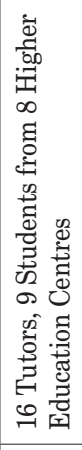 & 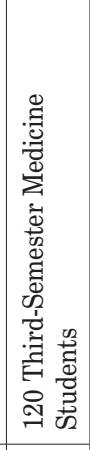 & 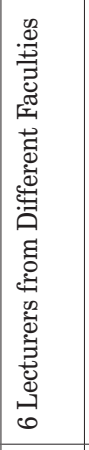 & 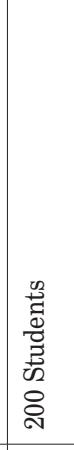 & 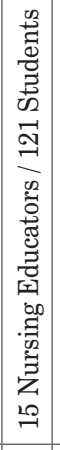 & 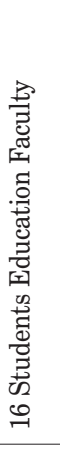 & 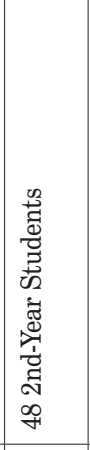 & 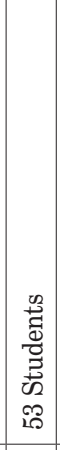 & 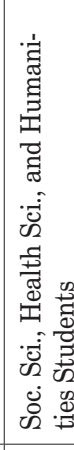 \\
\hline 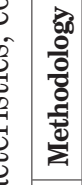 & 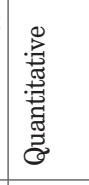 & 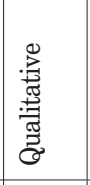 & 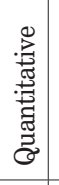 & 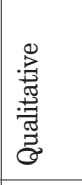 & 芠 & 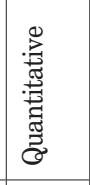 & 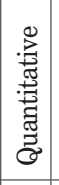 & 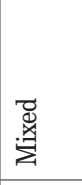 & 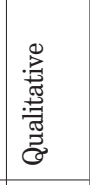 & & 芠 & 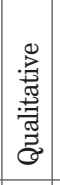 & 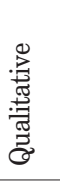 & 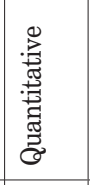 & 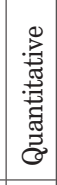 & \\
\hline
\end{tabular}

要

苞

용

产

है

$\stackrel{\infty}{\infty}$

要

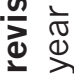

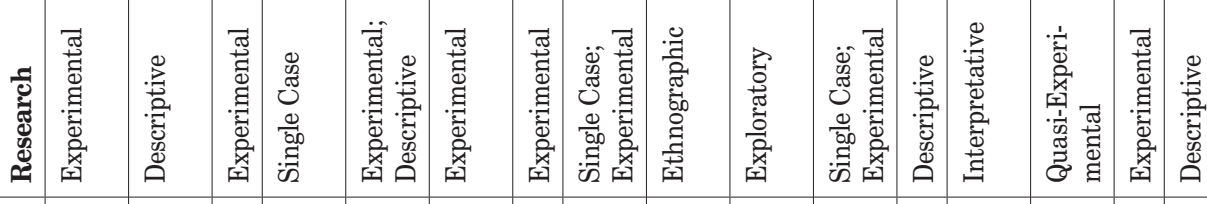

\begin{tabular}{|c|c|c|c|c|c|c|c|c|c|c|c|c|c|c|c|c|}
\hline 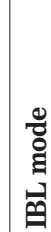 & 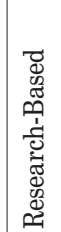 & 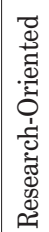 & 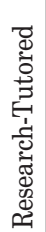 & 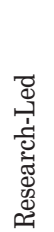 & 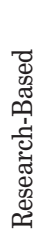 & 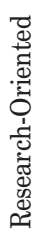 & 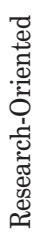 & 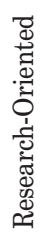 & 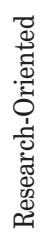 & 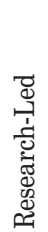 & 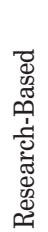 & 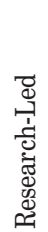 & 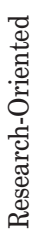 & 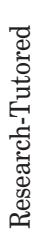 & 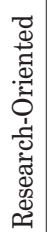 & 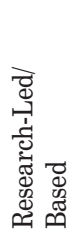 \\
\hline
\end{tabular}

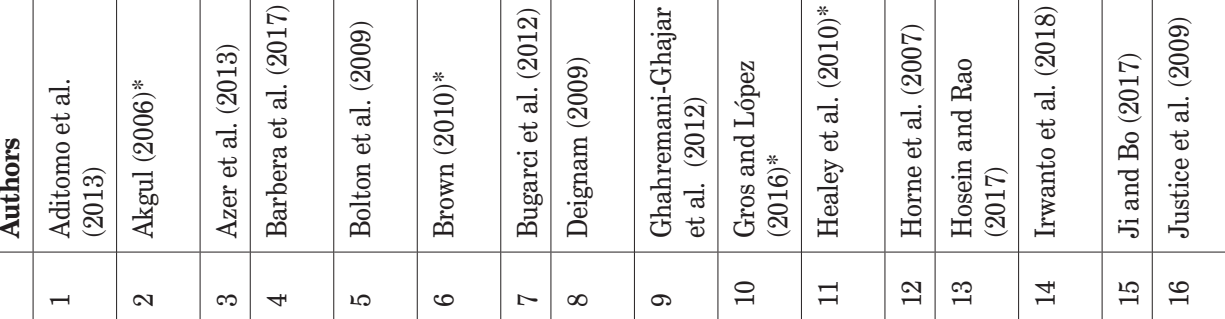




\begin{tabular}{|c|c|c|c|c|c|c|c|c|c|c|c|c|c|c|c|c|}
\hline \multirow[b]{2}{*}{ 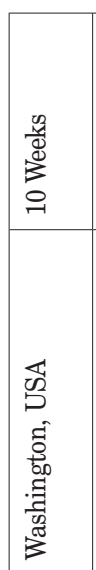 } & 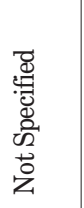 & 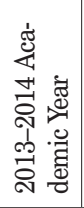 & 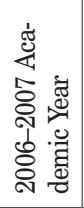 & 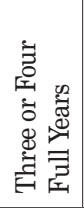 & 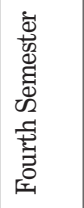 & 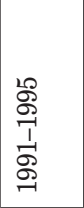 & 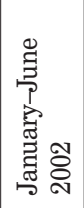 & 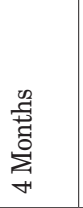 & 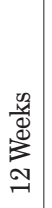 & 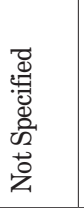 & 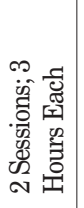 & 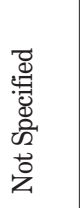 & 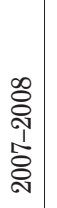 & 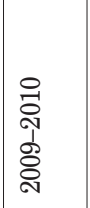 & & \\
\hline & 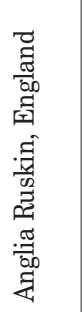 & 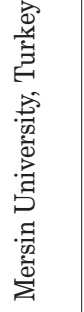 & 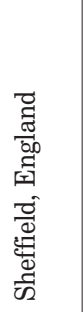 & 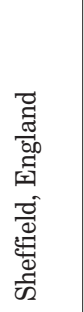 & 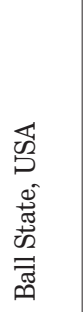 & 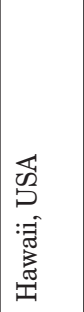 & 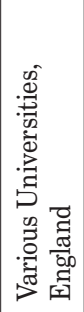 & 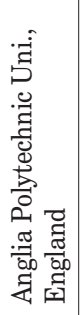 & 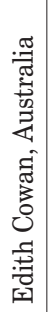 & 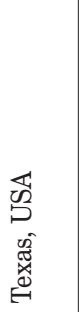 & 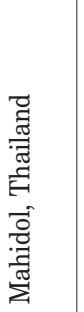 & 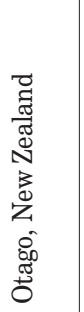 & 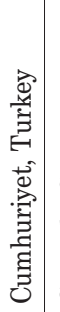 & 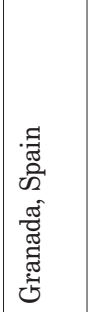 & & \\
\hline 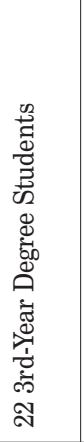 & 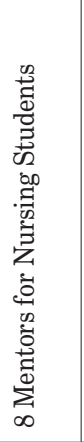 & 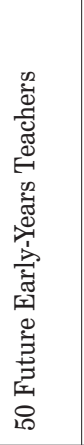 & 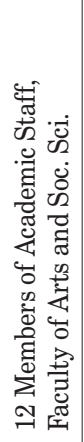 & 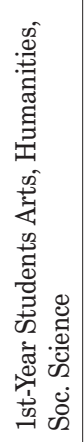 & 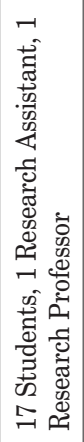 & 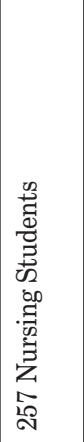 & 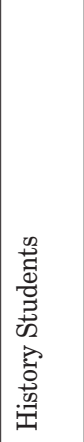 & 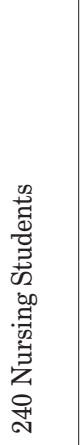 & 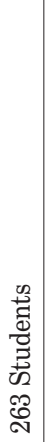 & 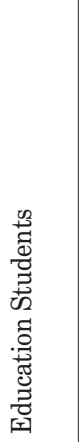 & 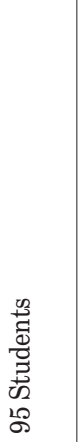 & 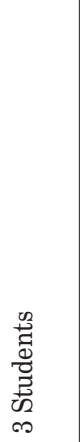 & 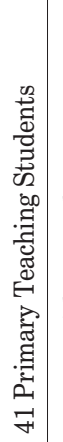 & 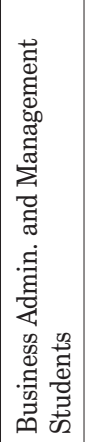 & 这 & 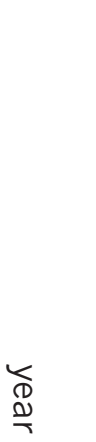 \\
\hline 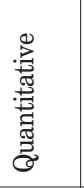 & 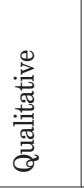 & 兑 & 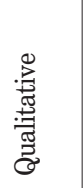 & 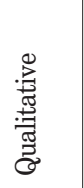 & 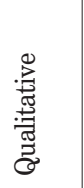 & 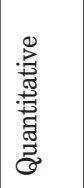 & 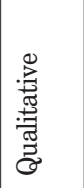 & 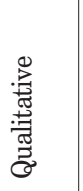 & 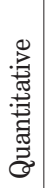 & 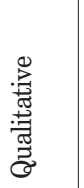 & 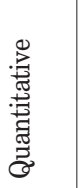 & 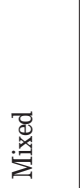 & 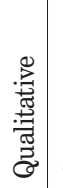 & 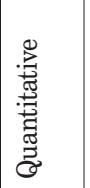 & & $\begin{array}{l}\infty \\
J \\
N \\
y \\
v\end{array}$ \\
\hline 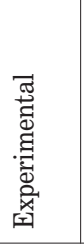 & 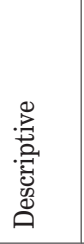 & 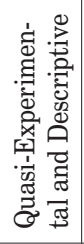 & 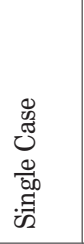 & 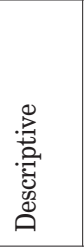 & 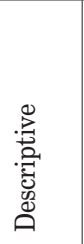 & 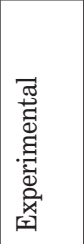 & 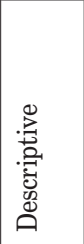 & 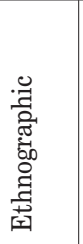 & 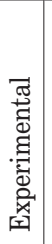 & 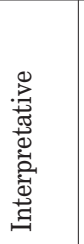 & 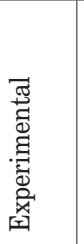 & 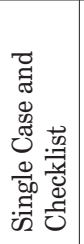 & 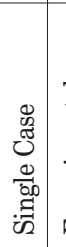 & 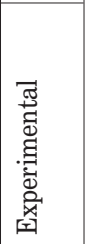 & 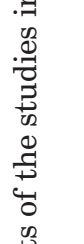 & 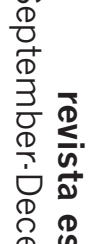 \\
\hline 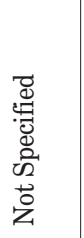 & 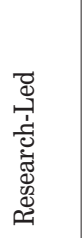 & 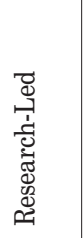 & 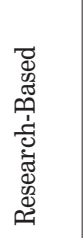 & 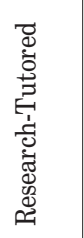 & 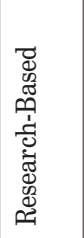 & 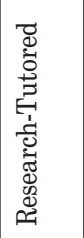 & 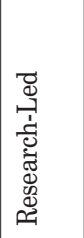 & 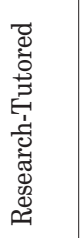 & 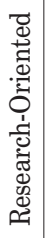 & 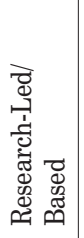 & 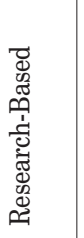 & 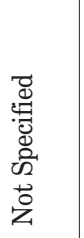 & 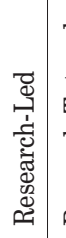 & 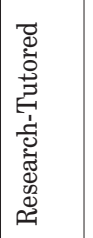 & 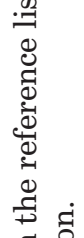 & 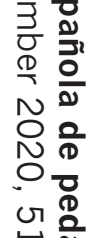 \\
\hline 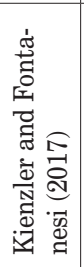 & 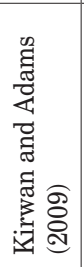 & 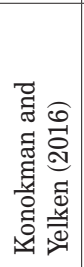 & 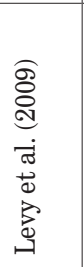 & 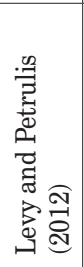 & 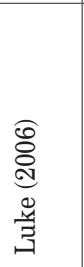 & 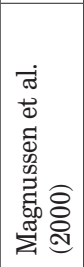 & 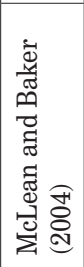 & 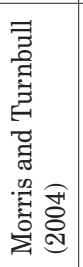 & 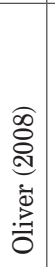 & 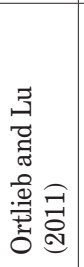 & 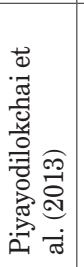 & 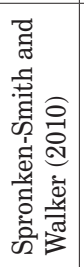 & 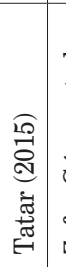 & 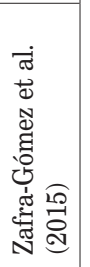 & 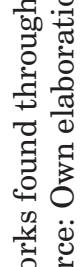 & 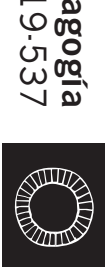 \\
\hline 드 & $\stackrel{\infty}{\sim}$ & $\stackrel{ }{2}$ & 요 & $\vec{N}$ & N & શึ & 今゙ & $\stackrel{19}{N}$ & $\stackrel{\mathscr{N}}{ }$ & స & $\stackrel{\infty}{\sim}$ & న্ণ & ฉి & $\vec{m}$ & $\begin{array}{l}3 \\
* \\
\text { \% }\end{array}$ & \\
\hline
\end{tabular}


Hosein and Rao (2017), Levy and Petrulis (2012), McLean and Barker (2004), and Ortlieb and Lu (2011) carried out descriptive and interpretative studies in which they analysed reflective essays and interviews using the ATLAS.ti program.

c) Mixed methodologies. Of these, $20 \%$ combine an experimental and interpretative method; $40 \%$ a single-case study and an experimental study; $20 \%$ a quasi-experimental study and an interpretative study; and $20 \%$ a single-case study and a checklist to extract quantitative data. In the study by Spronken-Smith and Walker (2010), three lecturers who used the IBL methodology were given a checklist about the focus of the inquiry, they were observed during class sessions, and they were interviewed about the processes and the results obtained.

\subsection{IBL Mode}

The studies display the four IBL modes described by Griffiths (2004) and Healey (2005) (see Table 1). Of the studies analysed, 29\% used the research-led mode, emphasising the elaboration of a knowledge-construction process dominated by the interests of the institution; $26 \%$ of the studies used the research-oriented mode, centring the students' learning process on research and on how knowledge is created; $26 \%$ of the studies used the research-based mode, as the teaching process focussed on the active role of the student in this process, minimising the role of lecturers; and 19\% were based on the research-tutored mode, as the teaching process focusses on small discussion groups guided by the lecturers who offer students feedback on the progress they make.

After identifying the modes in each of the studies analysed, the question arose of whether they all conceptualised the IBL methodology in the same way. Many authors regard the IBL methodology as a constructivist teaching method that connects students with learning, enabling them to explore research and knowledge creation from different perspectives (Healey, Jordan, Pell, \& Short, 2010; Levy \& Petrulis, 2012; Spronken-Smith \& Walker, 2010; Zafra-Gómez et al., 2015).

Although the terms used to describe the four IBL modes vary, they do all describe the students' participation in an inductive way in which they are encouraged to take responsibility for their own learning and knowledge exchange when working in groups. Some authors identify the IBL methodology with the problem-based learning methodology without distinguishing between them; both are regarded as part of a common philosophical approach to achieving inductive learning (Azer, Hasanato, Al-Nassar, Somily, \& AlSaadi, 2013; Deignan, 2009; Horne et al., 2007; Kirwan \& Adams, 2009; Morris \& Turnbull, 2004).

According to Ghahremani-Ghajar et al. (2012), IBL starts from a broad teaching-learning focus as it derives from a variety of interpretations and practices rooted in problem-based learning (PBL) that were originally proposed in medical education. Aditomo et al. (2013) note that IBL is based on pedagogical focuses 
aimed at achieving learning based on inquiry; in this case, it is applied through problem-based learning strategies, project-based learning, and case-based learning. Although there are differences with how IBL is conceptualised, including whether or not it is combined with other pedagogical focuses, all of the authors identify their methodology as an opportunity to achieve inductive learning, as: a) it enables responsibility-taking in learning and in actively contributing to the teach- ing process; and b) it offers multiple benefits in the training of future education and health professionals (Hosein \& Rao, 2017; Ji \& Bo, 2017; Magnussen, Ishida, \& Itano, 2000; Oliver, 2008).

\subsection{Student objectives and effects on students of using IBL}

In the studies analysed, five objectives in the implementation of IBL were identified, as well as the effects this methodology had on university students (Table 2).

TABLE 2. Effects on students of using IBL.

\begin{tabular}{|c|c|c|c|c|c|c|c|c|}
\hline & Authors and Year & $\begin{array}{l}\text { Increase in } \\
\text { Knowledge }\end{array}$ & $\begin{array}{l}\text { Search } \\
\text { Skills }\end{array}$ & $\begin{array}{c}\text { Academic } \\
\text { Perfor- } \\
\text { mance }\end{array}$ & $\begin{array}{l}\text { Self-Con- } \\
\text { fidence }\end{array}$ & $\begin{array}{c}\text { Self- } \\
\text { Learning }\end{array}$ & Motivation & $\begin{array}{l}\text { Critical } \\
\text { Thinking }\end{array}$ \\
\hline 1 & Aditomo et al. (2013) & Yes & Yes & - & - & Yes & Yes & Yes \\
\hline 2 & Akgul (2006) & Yes & Yes & - & - & Yes & - & - \\
\hline 3 & Azer et al. (2013) & Yes & Yes & Yes & Yes & Yes & Yes & - \\
\hline 4 & Barbera et al. (2017) & Yes & - & - & - & Yes & Yes & - \\
\hline 5 & Bolton et al. (2009) & Yes & - & - & - & Yes & Yes & Yes \\
\hline 6 & Brown (2010) & Yes & Yes & - & Yes & - & - & Yes \\
\hline 7 & Bugarci et al. (2012) & Yes & Yes & - & - & Yes & - & - \\
\hline 8 & Deignam (2009) & Yes & Yes & Yes & - & Yes & Yes & - \\
\hline 9 & $\begin{array}{l}\text { Ghahremani-Ghajar et } \\
\text { al. (2012) }\end{array}$ & Yes & - & - & - & Yes & - & Yes \\
\hline 10 & Gros and López (2016) & Yes & - & - & - & Yes & - & - \\
\hline 11 & Healey et al. $(2010)^{*}$ & Yes & Yes & - & - & Yes & Yes & - \\
\hline 12 & Horne et al. (2007) & Yes & Yes & - & Yes & - & Yes & Yes \\
\hline 13 & Hosein and Rao (2017) & Yes & Yes & - & Yes & Yes & Yes & - \\
\hline 14 & Irwanto et al. (2018) & Yes & Yes & Yes & - & Yes & - & Yes \\
\hline 15 & Ji and Bo (2017) & Yes & Yes & - & - & Yes & Yes & - \\
\hline 16 & Justice et al. (2009) & Yes & - & Yes & - & Yes & Yes & - \\
\hline 17 & $\begin{array}{l}\text { Kienzler and Fontanesi } \\
(2017)\end{array}$ & Yes & Yes & - & Yes & Yes & Yes & Yes \\
\hline 18 & $\begin{array}{l}\text { Kirwan and Adams } \\
(2009)\end{array}$ & Yes & - & - & Yes & Yes & Yes & - \\
\hline 19 & $\begin{array}{l}\text { Konokman and Yelken } \\
(2016)\end{array}$ & Yes & Yes & Yes & - & Yes & Yes & - \\
\hline 20 & Levy et al. (2009) & Yes & Yes & - & - & Yes & Yes & - \\
\hline
\end{tabular}


Lidia E. SANTANA-VEGA, Arminda SUÁREZ-PERDOMO and Luis FELICIANO-GARCÍA

\begin{tabular}{|c|c|c|c|c|c|c|c|c|}
\hline 21 & $\begin{array}{l}\text { Levy and Petrulis } \\
\text { (2012) }\end{array}$ & Yes & - & Yes & Yes & Yes & Yes & Yes \\
\hline 22 & Luke (2006) & Yes & - & Yes & - & Yes & - & - \\
\hline 23 & Magnussen et al. (2000) & Yes & - & - & - & - & Yes & Yes \\
\hline 24 & $\begin{array}{l}\text { McLean and Baker } \\
(2004)\end{array}$ & Yes & - & - & - & Yes & Yes & Yes \\
\hline 25 & $\begin{array}{l}\text { Morris and Turnbull } \\
\text { (2004) }\end{array}$ & Yes & - & - & Yes & Yes & Yes & - \\
\hline 26 & Oliver (2008) & Yes & Yes & - & Yes & Yes & Yes & - \\
\hline 27 & Ortlieb and Lu (2011) & Yes & Yes & - & Yes & Yes & Yes & Yes \\
\hline 28 & $\begin{array}{l}\text { Piyayodilokchai et al. } \\
\text { (2013) }\end{array}$ & Yes & Yes & Yes & - & Yes & Yes & - \\
\hline 29 & $\begin{array}{l}\text { Spronken-Smith and } \\
\text { Walker (2010) }\end{array}$ & Yes & Yes & - & - & Yes & Yes & - \\
\hline 30 & Tatar (2015) & Yes & Yes & - & - & Yes & Yes & - \\
\hline 31 & $\begin{array}{l}\text { Zafra-Gómez et al. } \\
\text { (2015) }\end{array}$ & Yes & - & Yes & Yes & Yes & Yes & Yes \\
\hline
\end{tabular}

Source: Own elaboration.

Objective 1. Examining the effect of using the IBL methodology in the teachinglearning process. Of the studies, $33 \%$ set out to evaluate what the main effects of implementing the IBL methodology were. For example, analysing students' misgivings about the implementation of IBL for preparing digital stories, or the use of alternative measures taking Pierce's triadic model of inference as a starting point (Konokman \& Yelken, 2016; Ortlieb \& Lu, 2011); observing how students accept information about the current economic and financial situation after working on the topic (Zafra-Gómez et al., 2015); examining possible departmental resistance to the implementation of IBL and the benefits it provides for graduates (Justice et al., 2009). The studies showed: 1) positive effects in the adoption of the IBL methodology, so long as the process is appropriately structured; 2) a significant increase in students' knowledge and skills in the short and long term; 3) an improvement in their academic performance (Azer et al., 2013; Konokman \& Yelken, 2016; Justice et al., 2009). The results of the study by Levy et al. (2009) show the great potential of the IBL methodology, as it offers opportunities for reflection and discussion that foster a high degree of empowerment; to do this, teaching staff need to have access to a wide range of IBL experiences, some structured and others led by the students.

The students identified the need to take the social aspect of IBL into account, as they learnt from the efforts of their classmates as well as their own efforts. Although they were aware of a possible increase in anxiety and stress in the learning process, they viewed it positively owing to the benefits for their learning (Deignan, 2009; Ji \& Bo, 2017; Luke, 2006). Healey et al. (2010) observed that IBL fosters students' interest in curriculum content and increases their motivation as they believe that pursuing postgraduate study is practical. 
Objective 2. Evaluating the development of research competences in students. Of these studies, $11 \%$ set out to examine students' own awareness of the development of their research competences when writing reflective essays (Hosein \& Rao, 2017), and to evaluate how the inquiry based teaching process is understood and experienced, relating it to the students' epistemological construction (Levy \& Petrulis, 2012). These studies showed a growing enthusiasm among students for research learning, as they see the potential of a student-centred methodology for their future training (Hosein \& Rao, 2017; Levy \& Petrulis, 2012). Akgul (2006) observed that students regarded science as a process of finding more truths or facts, and that their levels of learning depended on their involvement and commitment.

Objective 3. Promoting learning tasks or results by implementing $I B L$. Of these studies, $22 \%$ use IBL as a way of introducing values related to volunteering in a non-profit organisation (Bolton, Brennan, \& Terry, 2009), or of investigating students' achievement when a cyclical learning model is assumed that is complemented by multimedia resources (Piyayodilokchai et al., 2013). We observed that this methodology is effective for inverting the role of the student as a mere passive receptor; students were able to learn how learning is managed, they accepted challenges, and their self-confidence increased (Bolton et al., 2009; Kienzler \& Fontanesi, 2017). Aditomo et al. (2013) identify eight research tasks for achieving satisfactory learning outcomes after applying IBL: academic research, simplified research, inquiry-based literature, inquiry-based discussion, applied research, simulated applied research, implementing practice, and role playing. After implementing IBL, a wide range of educational objectives were achieved that involved cognitive, metacognitive, affective, social, and epistemological aspects.

Objective 4. Exploring the development of critical thinking. Of these studies, $19 \%$ set out to explore what the students' critical perspectives were when a research challenge was posed in language learning (Ghahremani-Ghajar et al., 2012), or to examine changes in students' critical thinking after implementing IBL, comparing their opinion at the start of their course with their opinion at the end of it (Magnussen et al., 2000; Tatar, 2015). It was apparent that if the IBL methodology was well structured and implemented in small groups, it provided benefits for university students, and a significant impact on critical thinking and problemsolving skills was also noted (Irwanto et al., 2018; Ghahremani-Ghajar et al., 2012; Tatar, 2015; Gros \& López, 2016). Bugarcic, Zimbardi, Macaranas, and Thorn (2012) observed that using IBL promotes meaningful learning and encourages students to approach ideas and critically evaluate what they encounter in a "real" research setting, while at the same time obtaining a high level of detailed knowledge of the content.

Objective 5. Promoting personal skills and competences for self-learning. Of the studies, $15 \%$ had the aim of examining whether using IBL has an impact on mo- 
tivation, self-confidence, self-learning, scientific attitudes, and engagement in participation (Ji \& Bo, 2017; Oliver, 2008; Brown, 2016), or examining students' capacity for autonomy in their learning, positively and/or negatively, and their reactions and interpretations in the face of cyclical research (Luke, 2006). The students reported that the IBL methodology stimulated their desire for knowledge, stimulated their enthusiasm for self-learning, and strengthened their self-efficacy and motivation.

In general, studies identify: a) increased student motivation, b) improved understanding of subjects and of their relevance to society, c) increased collaboration between students when working together to achieve a common goal, d) an increase in joint responsibility in carrying out tasks, e) improved interpersonal skills and skills in performing work roles (Bruder \& Prescott, 2013; Frezell, 2018). One hundred per cent of the studies showed that using IBL fostered increased knowledge by students; $61 \%$ demonstrated a development of research skills; $29 \%$ showed an increase in academic performance; $36 \%$ an increase in students' self-confidence; 90\% an increase in students' self-learning; $70 \%$ enhanced motivation for learning; and $40 \%$ an increase in critical thinking.

\subsection{Some limitations of the studies}

This section sets out some limitations of the studies:

1) The research in the articles was carried out on the basis of a particular IBL model and with very specific groups of students; although a snapshot of the students' experience and of facilitator perspectives in a given context was obtained, the application of IBL with other samples should be explored to consider generalising the results (Horne et al., 2007; SpronkenSmith \& Walker, 2010).

2) In some studies, the limitations derive from the university structures, as there can be resistance in departments as they do not see obvious benefits in the implementation of IBL or they believe that it might alter existing power structures and how resources are assigned (Justice, Rice, Roy, Hudspith, \& Jenkins, 2009).

3) Other limitations derive from the research method used; using a qualitative method risks biasing students' responses towards an excessively optimistic focus on IBL if the interviews are carried out by the principal researcher (Luke, 2006). The use of focus groups can influence results as students who enjoyed the class or interacted with the teacher-researcher sometimes talk more (Luke, 2006; Morris \& Turnbull, 2004). Regarding the quantitative methodology, standardised measurement instruments can bypass the experiences and emotions generated in the process by only offering students closed answers (Magnussen et al., 2000).

\section{Conclusions}

This systematic review identified 31 articles on IBL experiences in social science and health science courses. These experiences were not limited to a single country and they approached the appli- 
cation of IBL from different research perspectives (quantitative, qualitative, and mixed). The objectives identified in the studies not only focus on establishing the possibility of implementing IBL in the classroom but also on understanding what the main effects are on the teaching process, what research competences the students acquire, how learning results are promoted, how critical thinking is developed, and how personal skills and competences are fostered. To discover whether this methodology can be beneficial for the teaching-learning process and the development of students' research competences, the authors applied different IBL modes and determined their strengths and weaknesses. As strengths, the authors identified: exploring knowledge in greater depth, promoting cooperative learning, students' commitment in their self-learning, and increased critical thinking. Among the weaknesses, the following were noted: the inability to cover the students' learning expectations and university hierarchies' misgivings about IBL.

IBL favours meaningful learning by university students by involving them in a process of doing research and it strengthens the inquiry-teaching bond so long as the process allows agents to express their experiences and emotions. To carry out IBL processes, it is necessary to emphasise to students the need to construct knowledge and strengthen their responsibility in the teaching-learning process (Healey, 2005; Levy \& Petrulis, 2012). The IBL methodology provides a strong social scaffolding and active learning, fostering per- sonal research skills, joint responsibility in completing tasks, and the capacity for reflection in learning situations (Bruder \& Prescott, 2013; Frezell, 2018; Hmelo-Silver et al., 2007).

In essence, IBL provides a learning process that: a) fosters the development of research competences in a student cohort that accepts the challenge of their self-learning; b) provides a space for knowledge creation stimulated by inquiry; and c) fosters students' interest in and commitment to their learning process and doing work of high academic quality.

This study's main limitations are that it restricted its search to: 1) articles on IBL written in English, 2) articles that applied this methodology to qualifications from the social sciences and health sciences in a university setting. In future research, it will be necessary to expand the search criteria to include works written in Spanish and works that cover other fields of knowledge and educational stages. Nonetheless, this study provides valuable information for identifying possibilities for implementing IBL in qualifications that educate future professionals in the fields of the social sciences and health sciences.

\section{References}

Aditomo, A., Goodyear, P., Bliuc, A. M., \& Ellis, R. A. (2013). Inquiry-based learning in higher education: principal forms, educational objectives, and disciplinary variations. Studies in Higher Education, 38 (9), 1239-1258. doi: https://doi.org/10.1080/03075079.2011.616584

Åkerlind, G. S. (2008). An academic perspective on research and being a researcher: an 
integration of the literature. Studies in Higher Education, 33 (1), 17-31. doi: https://doi. org/10.1080/03075070701794775

Akgul, E. M. (2006). Teaching science in an inquiry-based learning environment: What it means for pre-service elementary science teachers. Eurasia Journal of Mathematics, Science and Technology Education, 2 (1), 71-81. doi: https://doi.org/10.12973/ejmste/75439

Azer, S. A., Hasanato, R., Al-Nassar, S., Somily, A., \& AlSaadi, M. M. (2013). Introducing integrated laboratory classes in a PBL curriculum: impact on student's learning and satisfaction. BMC Medical Education, 13 (1), 71. doi: https://doi.org/10.1186/1472-6920-13-71

Barbera, E., García, I., \& Fuertes-Alpiste, M. (2017). A Co-Design Process Microanalysis: Stages and Facilitators of an Inquiry-Based and Technology-Enhanced Learning Scenario. The International Review of Research in Open and Distributed Learning, 18 (6), 105-125. doi: https://doi.org/10.19173/irrodl.v18i6.2805

Bevins, S., \& Price, G. (2016). Reconceptualising inquiry in science education. International Journal of Science Education, 38 (1), 17-29. doi: https://doi.org/10.1080/09500693.2015.1124300

Bolton, E. B., Brennan, M. A., \& Terry, B. D. (2009). Students Learn How Nonprofits Utilize Volunteers through Inquiry-Based Learning. International Journal of Teaching and Learning in Higher Education, 21 (3), 285-294.

Brew, A. (2003). Teaching and research: New relationships and their implications for inquiry-based teaching and learning in higher education. Higher Education Research \& Development, 22 (1), 3-18. doi: https://doi. org $/ 10.1080 / 0729436032000056571$

Brown, J. A. (2016). Evaluating the effectiveness of a practical inquiry based learning bioinformatics module on undergraduate student engagement and applied skills. Biochemistry and molecular biology education, 44 (3), 304-313. doi: https://doi.org/10.1002/bmb.20954

Bruder, R., \& Prescott, A. (2013). Research evidence on the benefits of IBL. ZDM Mathematics Education, 45 (6), 811-822. https://doi. org/10.1007/s11858-013-0542-2

Bugarcic, A., Zimbardi, K., Macaranas, J., \& Thorn, P. (2012). An inquiry based practical for a large, foundation level undergraduate laboratory that enhances student understanding of basic cellular concepts and scientific experimental design. Biochemistry and Molecular Biology Education, 40 (3), 174-180. doi: https://doi. org $/ 10.1002 / \mathrm{bmb} .20587$

Deignan, T. (2009). Enquiry-Based Learning: Perspectives on practice. Teaching in Higher Education, 14 (1), 13-28. doi: https://doi. org/10.1080/13562510802602467

Frezell, D. (2018). Impact of Inquiry Based Learning on Students' Motivation, Engagement and Attitude in Science. Electronic Theses and Dissertations, 7356. Retrieved from https://scholar.uwindsor.ca/etd/7356 (Consulted on 2019-11-18).

Ghahremani-Ghajar, S. S., Mohammadi Doostdar, H., \& Sadegh Mirhosseini, S. M. (2012). We have been living with this pain: Enquiry-based language learning in Iranian higher education. Teaching in Higher Education, 17 (3), 269-281. doi: https://doi.org/10.1080/13562517.2011.611 864

Griffiths, R. (2004). Knowledge production and the research-teaching nexus: The case of the built environment disciplines. Studies in Higher Education, 29 (6), 709-726. doi: https://doi. org $/ 10.1080 / 0307507042000287212$

Gros, B., \& López, M. (2016). Students as cocreators of technology-rich learning activities in higher education. International Journal of Educational Technology in Higher Education, 13 (1), 28. doi: https://doi.org/10.1186/s41239016-0026-x

Healey, M. (2005). Linking research and teaching exploring disciplinary spaces and the role of inquiry-based learning. In R. Barnett (Coord.), Reshaping the university: New relationships between research, scholarship and teaching (pp. 67-78). Buckingham: Open University Press.

Healey, M., \& Jenkins., A. (2009). Developing undergraduate research and inquiry. Research Report to the Higher Education Academy. York: Higher Education Academy.

Healey, M., Jordan, F., Pell, B., \& Short, C. (2010). The research-teaching nexus: a case study of students' awareness, experiences and perceptions of research. Innovations in Education and 
Teaching International, 47 (2), 235-246. doi: https://doi.org/10.1080/14703291003718968

Herman, W. E., \& Pinard, M. R. (2015). Critical-

ly Examining inquiry-based learning: John Dewey in theory, history, and practice. In Inquiry-Based Learning For Multidisciplinary Programs: A Conceptual and Practical Resource for Educators (pp. 43-62). Bingley, Yorkshire: Emerald Group Publishing Limited.

Hmelo-Silver, C. E., Duncan, R. G., \& Chinn, C. A. (2007). Scaffolding and achievement in problem-based and inquiry learning: a response to Kirschner, Sweller, and Clark. Educational Psychologist, 42 (2), 99-107. doi: https://doi. org $/ 10.1080 / 00461520701263368$

Horne, M., Woodhead, K., Morgan, L., Smithies, L., Megson, D., \& Lyte, G. (2007). Using enquiry in learning: From vision to reality in higher education. Nurse Education Today, 27 (2), 103-112. doi: https://doi.org/10.1016/j.nedt.2006.03.004

Hosein, A., \& Rao, N. (2017). Students' reflective essays as insights into student centred-pedagogies within the undergraduate research methods curriculum. Teaching in Higher Education, 22 (1), 109-125. doi: https://doi.org/10.1080/135 62517.2016.1221804

Hunter, A. B., Laursen, S. L., \& Seymour, E. (2007). Becoming a scientist: The role of undergraduate research in students' cognitive, personal, and professional development. Science Education, 91 (1), 36-74. doi: https://doi.org/10.1002/ sce. 20173

Irwanto, S., Saputro, A. D., \& Prodjosantoso, A. K. (2018). Promoting Critical Thinking and Problem Solving Skills of Preservice Elementary Teachers through Process-Oriented Guided-Inquiry Learning (POGIL). International Journal of Instruction, 11 (4), 777-794. doi: https:// doi.org/10.12973/iji.2018.11449a

Ji, F., \& Bo, H. (2017). Function Design for Inquiry-Based Learning Platform Based on a Computer Network. International Journal of Emerging Technologies in Learning (iJET), 12 (02), 40-51. doi: https://doi.org/10.3991/ijet. v12i02.6043

Justice, C., Rice, J., Roy, D., Hudspith, B., \& Jenkins, H. (2009). Inquiry-based learning in higher education: administrators' perspectives on integrating inquiry pedagogy into the cur- riculum. Higher Education, 58 (6), 841. doi: https://doi.org/10.1007/s10734-009-9228-7

Justice, C., Rice, J., \& Warry, W. (2009). Developing Useful and Transferable Skills: Course Design to Prepare Students for a Life of Learning. International Journal for the Scholarship of Teaching and Learning, 3 (2), 1-19. doi: https://doi. org/10.20429/ijsotl.2009.030209

Justice, C., Rice, J., Warry, W., Inglis, S., Miller, S., \& Sammon, S. (2007). Inquiry in higher education: Reflections and directions on course design and teaching methods. Innovative Higher Education, 31 (4), 201-214. doi: https://doi. org/10.1007/s10755-006-9021-9

Kahn, P. y O'Rourke, K. (2004). Guide to curriculum design: Enquiry-based learning. Retrieved from https://www.academia.edu/460509/Guide_to_Curriculum_Design_Enquiry-Based_ Learning (Consulted on 2019-11-26).

Kienzler, H., \& Fontanesi, C. (2017). Learning through inquiry: A global health hackathon. Teaching in Higher Education, 22 (2), 129-142. doi: https://doi.org/10.1080/13562517.2016.122 1805

Kirwan, A., \& Adams, J. (2009). Students' views of enquiry-based learning in a continuing professional development module. Nurse Education Today, 29 (4), 448-455. doi: https://doi. org/10.1016/j.nedt.2008.09.003

Konokman, Y., \& Yelken, T. (2016). Preparing Digital Stories through the Inquiry-Based Learning Approach: Its Effect on Prospective Teachers' Resistive Behaviors toward Research and Technology-Based Instruction. Educational Sciences: Theory and Practice, 16 (6), 2141-2165. doi: https://doi.org/10.12738/ estp.2016.6.0410

Levy, P., Aiyegbayo, O., \& Little, S. (2009). Designing for inquiry based learning with the Learning Activity Management System. Journal of Computer Assisted Learning, 25 (3), 238-251. doi: https://doi.org/10.1111/j.13652729.2008.00309.x

Levy, P., \& Petrulis, R. (2012). How do first-year university students experience inquiry and research, and what are the implications for the practice of inquiry-based learning? Studies in Higher Education, 37 (1), 85-101. doi: https:// doi.org/10.1080/03075079.2010.499166 
Liberati, A., Altman, D. G., Tetzlaff, J., Mulrow, C., Gøtzsche, P. C., Ioannidis, J. P., .... \& Moher, D. (2009). The PRISMA statement for reporting systematic reviews and meta-analyses of studies that evaluate health care interventions: explanation and elaboration. PLoS medicine, 6 (7), e1000100. doi: https:// doi.org/10.1371/journal.pmed.1000100

Luke, C. L. (2006). Fostering Learner Autonomy in a Technology Enhanced, Inquiry Based Foreign Language Classroom. Foreign Language Annals, 39 (1), 71-86. doi: https://doi. org/10.1111/j.1944-9720.2006.tb02250.x

Maass, K., \& Engeln, K. (2018). Effects of Scaledup Professional Development Courses About Inquiry-Based Learning on Teachers. Journal of Education and Training Studies, 6 (4), 1-16. doi: https://doi.org/10.11114/jets. v6i4.3083

Magnussen, L., Ishida, D., \& Itano, J. (2000). The impact of the use of inquiry-based learning as a teaching methodology on the development of critical thinking. Journal of Nursing Education, 39 (8), 360-364. doi: https://doi. org/10.3928/0148-4834-20001101-07

McLean, M., \& Barker, H. (2004). Students making progress and the 'research teaching nexus' debate. Teaching in Higher Education, 9 (4), 407-419. doi: https://doi. org $/ 10.1080 / 1356251042000252354$

Minner, D. D., Levy, A. J., \& Century, J. (2010). Inquiry based science instruction-what is it and does it matter? Results from a research synthesis years 1984 to 2002. Journal of Research in Science Teaching: The Official Journal of the National Association for Research in Science Teaching, 47 (4), 474-496. doi: https://doi.org/10.1002/tea.20347

Morris, D., \& Turnbull, P. (2004). Using student nurses as teachers in inquiry-based learning. Journal of Advanced Nursing, 45 (2), 136-144. doi: https://doi.org/10.1046/j.13652648.2003.02875.x

Oliver, R. (2008). Engaging first year students using a web-supported inquiry-based learning setting. Higher Education, 55 (3), 285-301. doi: https://doi.org/10.1007/s10734-007-9055-7

Ortlieb, E., \& Lu, L. (2011). Improving teacher education through inquiry-based learning.
International Education Studies, 4 (3), 41-46. doi: https://doi.org/10.5539/ies.v4n3p41

Piyayodilokchai, H., Panjaburee, P., Laosinchai, P., Ketpichainarong, W., \& Ruenwongsa, P. (2013). A 5E Learning Cycle ApproachBased, Multimedia-Supplemented Instructional Unit for Structured Query Language. Journal of Educational Technology \& Society, 16 (4), 146-159.

Prince, M., \& Felder, R. (2007). The many faces of inductive teaching and learning. Journal of College Science Teaching, 36 (5), 14.

Spronken-Smith, R., Angelo, T., Matthews, H., O'Steen, B., \& Robertson, J. (2007). How effective is inquiry-based learning in linking teaching and research. In An International Colloquium on International Policies and Practices for Academic Enquiry (pp. 19-21). Winchester, UK: Marwell.

Spronken-Smith, R., \& Walker, R. (2010). Can inquiry based learning strengthen the links between teaching and disciplinary research? Studies in Higher Education, 35 (6), 723-740. doi: https:/doi. org $/ 10.1080 / 03075070903315502$

Tatar, N. (2015). Pre-service teachers' beliefs about the image of a science teacher and science teaching. Journal of Baltic Science Education, 14 (1), 34-44.

Zafra-Gómez, J. L., Román-Martínez, I., \& Gómez-Miranda, M. E. (2015). Measuring the impact of inquiry-based learning on outcomes and student satisfaction. Assessment \& Evaluation in Higher Education, 40 (8), 1050-1069. doi: https://doi.org/10.1080/0260 2938.2014.963836

\section{Authors' biographies}

Lidia E. Santana-Vega has a doctorate in Philosophy and Educational Science and a degree in Psychology. She holds a university chair in the Department of Teaching and Educational Research at the Universidad de La Laguna. She is coordinator of the GIOES consolidated research group. Her research interests focus on attention/support for 
students, the life design of young people at risk of social exclusion, and decision making and gender.

iD http://orcid.org/0000-0002-2543-6543

Arminda Suárez-Perdomo has a doctorate in Educational Psychology and is Assistant Professor in the Department of the Family, School, and Society at the Universidad Internacional de la Rioja (UNIR). Her research focusses on fostering positive parenting in virtual experiential learning situations and on parents' digital competence, as well as on the influence of procrastination on the academic performance of university students.

\section{iD} https://orcid.org/0000-0002-6755-5284

Luis Feliciano-García has a doctorate in Philosophy and Educational Sciences, and is Associate Professor in the Department of Teaching and Educational Research at the Universidad de La Laguna. He is a member of the GIOES research group. His research focusses on active methodologies and academic-employment decision making.

iD http://orcid.org/0000-0002-2909-4990 


\section{revista española de pedagogía año 78, n. ${ }^{\circ}$ 277, septiembre-diciembre 2020}

Spanish Journal of Pedagogy

year 78, n. 277, September-December 2020

Table of Contents

\section{Sumario}

\section{Mastering time and personal and social development \\ Dominio del tiempo y desarrollo personal y social}

Guest editors: Ana Ponce de León Elizondo, \& M. ${ }^{a}$

Ángeles Valdemoros San Emeterio

Editoras invitadas: Ana Ponce de León Elizondo y M. Ángeles

Valdemoros San Emeterio

Ana Ponce de León Elizondo, \& M. Ángeles

Valdemoros San Emeterio

Introduction: Mastering time and personal and social development

Presentación: Dominio del tiempo y desarrollo personal y social 373

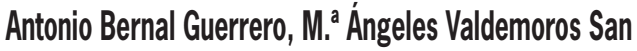

Emeterio, \& Alfredo Jiménez Eguizábal

Time, power, and education. Rethinking the

construction of personal identity and educational

policy decisions

Tiempo, poder y educación. Repensando la construcción de la

identidad personal y las decisiones de la politica educativa

José Antonio Caride

To educate and educate ourselves in time, pedagogically and socially

Educar y educarnos a tiempo, pedagógica y socialmente
Rosa Ana Alonso Ruiz, Magdalena Sáenz de Jubera Ocón, \& Eva Sanz Arazuri

Shared time between grandparents and

grandchildren: A time for personal development

Tiempos compartidos entre abuelos y nietos, tiempos de desarrollo personal

Nuria Codina, Rafael Valenzuela, \& José Vicente

Pestana

From the perception to the uses of time: Time

perspective and procrastination among adults in Spain

De la percepción a los usos del tiempo: perspectiva temporal y procrastinación de adultos en España

435

José Manuel Muñoz-Rodriguez, Patricia Torrijos Fincias, Sara Serrate González, \& Alicia Murciano Hueso

Digital environments, connectivity and education:

Time perception and management in the construction of young people's digital identity

Entornos digitales, conectividad y educación. Percepción y

gestión del tiempo en la construcción de la identidad digital de

la juventud

Ángel De-Juanas Oliva, Francisco Javier GarciaCastilla, \& Ana Ponce de León Elizondo

The time of young people in social difficulties: Use,

management, and socio-educational actions

El tiempo de los jóvenes en dificultad social: utilización,

gestión y acciones socioeducativas 


\section{Studies}

Estudios

Catherine L'Ecuyer, \& José Ignacio Murillo

Montessori's teleological approach to education and

its implications

El enfoque teleológico de la educación Montessori y sus

implicaciones

Lidia E. Santana-Vega, Arminda Suárez-Perdomo, \& Luis Feliciano-Garcia

Inquiry-based learning in the university context:

A systematic review

El aprendizaje basado en la investigación en el contexto universitario: una revisión sistemática

\section{Book reviews}

Moreno, A. (2020). Personalizar, un modelo para una educación de calidad en el siglo xx.. Informe Delphi de Expertos [Personalise, a model for quality education in the 21st century. Delphi Expert Report] (Cristina Medrano Pascual). Fuentes, J. L. (Ed.) (2019).

499 Ética para la excelencia educativa [Ethics for educational excellence] (Ana García-Bravo).

Table of contents of the year 2020 Índice del año 2020

551

This is the English version of the research articles and book reviews published originally in the Spanish printed version of issue 277 of the revista española de pedagogía. The full Spanish version of this issue can also be found on the journal's website http://revistadepedagogia.org.

ISSN: 0034-9461 (Print), 2174-0909 (Online)

https://revistadepedagogia.org/

Depósito legal: M. 6.020 - 1958

INDUSTRIA GRÁFICA ANZOS, S.L. Fuenlabrada - Madrid 